\title{
Hedgehog interacting protein-expressing lung fibroblasts suppress lymphocytic inflammation in mice
}

\author{
Jeong H. Yun, ${ }^{1,2,3}$ ChangHee Lee, ${ }^{3,4}$ Tao Liu, ${ }^{1}$ Siqi Liu, ${ }^{1}$ Edy Y. Kim, ${ }^{2,3}$ Shuang Xu, Jeffrey L. Curtis, ${ }^{5,6}$ \\ Luca Pinello, ${ }^{3,7}$ Russell P. Bowler, ${ }^{8}$ Edwin K. Silverman, ${ }^{1,3}$ Craig P. Hersh, ${ }^{1,2,3}$ and Xiaobo Zhou ${ }^{1,3}$ \\ ${ }^{1}$ Channing Division of Network Medicine, and 'Division of Pulmonary and Critical Care, Department of Medicine, \\ Brigham and Women's Hospital, Boston, Massachusetts, USA. ${ }^{3}$ Harvard Medical School, Boston, Massachusetts, USA. \\ ${ }^{4}$ Department of Genetics, Blavatnik Institute, Harvard Medical School, Boston, Massachusetts, USA. ${ }^{5}$ Department of \\ Internal Medicine, University of Michigan Medical School \& Michigan Medicine, Ann Arbor, Michigan, USA. VA Ann Arbor \\ Healthcare System, Ann Arbor, Michigan, USA. 'Department of Pathology, Massachusetts General Hospital, Boston, \\ Massachusetts, USA. ${ }^{8}$ Department of Medicine, National Jewish Health, Denver, Colorado, USA.
}

\begin{abstract}
Chronic obstructive pulmonary disease (COPD) is mainly caused by cigarette smoking and characterized by chronic inflammation in vulnerable individuals. However, it is unknown how genetic factors may shape chronic inflammation in COPD. To understand how hedgehog interacting protein, encoded by HHIP gene identified in the genome-wide association study in COPD, plays a role in inflammation, we utilized $\mathrm{Hhip}^{+/-}$mice that present persistent inflammation and emphysema upon aging similar to that observed in human COPD. By performing single-cell RNA sequencing of the whole lung from mice at different ages, we found that $\mathrm{Hhip}^{+/-}$mice developed a cytotoxic immune response with a specific increase in killer cell lectin-like receptor C1-positive CD8 ${ }^{+}$ T cells with upregulated $I f n \gamma$ expression recapitulating human COPD. Hhip expression was restricted to a lung fibroblast subpopulation that had increased interaction with $C D 8^{+} T$ lymphocytes in Hhip $^{+/-}$compared with $\mathrm{Hhip}^{+/+}$during aging. Hhip-expressing lung fibroblasts had upregulated IL-18 pathway genes in $\mathrm{Hhip}^{+/-}$lung fibroblasts, which was sufficient to drive increased levels of IFN- $\gamma$ in $\mathrm{CD8}^{+} \mathrm{T}$ cells ex vivo. Our finding provides insight into how a common genetic variation contributes to the amplified lymphocytic inflammation in COPD.
\end{abstract}

Authorship note: JHY and CL contributed equally to this work.

Conflict of interest: JHY has received personal fees from Bridge Biotherapeutics. JC has received personal fees from AstraZeneca and Novartis. $\mathrm{CPH}$ reports grants from Bayer, Boehringer Ingelheim, Novartis, and Vertex and personal fees from 23andMe. EKS has received institutional grant support from GlaxoSmithKline and Bayer. XZ has received institutional grant support from Bayer.

Copyright: (c) 2021, Yun et al. This is an open access article published under the terms of the Creative Commons Attribution 4.0 International License.

Submitted: September 23, 2020

Accepted: July 21, 2021

Published: August 10, 2021

Reference information: JCI Insight. 2021;6(17):e144575

https://doi.org/10.1172/jci.

insight.144575.

\section{Introduction}

Chronic obstructive pulmonary disease (COPD) is a highly prevalent chronic inflammatory lung disease characterized by progressive airflow limitation. Cigarette smoking is the major risk factor for COPD. However, there is marked heterogeneity in disease susceptibility and clinical manifestations of COPD among smokers with similar smoking histories. Genetic factors have been identified to account for these variations, but the cellular and molecular basis of genetic susceptibility has not been fully understood. Variable inflammation among smokers, including infiltration and activation of immune cells, possibly attributable to genetic risks of COPD, are amplified in patients with COPD compared with asymptomatic smokers

The hedgehog interacting protein (HHIP) locus on chromosome $4 \mathrm{q} 31$ is one of the most replicated loci, strongly associated with COPD in multiple genome-wide association studies (GWAS) (1-4). It has been associated with severity and distribution of emphysema $(5,6)$ as well as with lung function, measured as forced

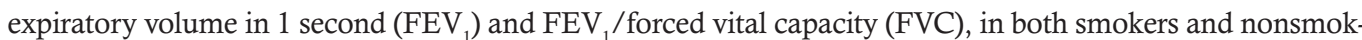
ers $(1,7,8)$. We have previously found COPD risk alleles at the HHIP locus to be associated with reduced distal enhancer activity, suggesting lower levels of HHIP increase the risk for COPD (9). HHIP negatively regulates the hedgehog signaling pathway by competitively binding with hedgehog ligands. Hhip-knockout mice develop hypoplastic lungs due to defective branching morphogenesis, leading to postnatal lethality (10). However, Hhip-haploinsufficient $\left(\right.$ Hhip $^{+/-}$) mice develop normal lungs, histologically indistinguishable from WT controls (11). We found that with an approximately 30\% reduction of HHIP mRNA levels in lungs, $H_{h i p^{+/-}}$mice display not only more severe emphysema, but also prominent airway lymphoid aggregates after chronic cigarette smoke (CS) exposure, characteristic of severe COPD (12). Furthermore, Hhip ${ }^{+/-}$mice form 
evident lymphoid aggregates as they age, even in the absence of CS exposure, suggesting that Hhip ${ }^{+/-}$mice have inherent susceptibility toward exaggerated inflammation, a key feature of human COPD lungs that was not recapitulated in other murine models of COPD (13). Although HHIP was shown to be expressed in mesenchyme during murine lung development (10), it remains unclear which cell types express HHIP after lung development and how haploinsufficiency of HHIP results in lymphocytic inflammation.

To address these questions, we investigated associations between HHIP variant and COPD phenotypes in participants in the COPDGene Study and assessed the impact of Hhip haploinsufficiency in murine lungs at different age points by single-cell RNA sequencing. Our study suggests that Hhip, expressed in murine lung fibroblasts, represses $\mathrm{CD}^{+} \mathrm{T}$ cell activation, possibly through IL-18. Insufficient levels of Hhip result in expansion of dysregulated and activated $\mathrm{CD} 8^{+} \mathrm{T}$ cells, contributing to lymphocytic inflammation. In human lungs, HHIP is expressed in both mesenchymal and epithelial compartments. Patients with COPD carrying the HHIP risk allele display elevated IL-18 levels and increased COPD exacerbation frequency. These findings provide mechanistic insights into chronic inflammation seen in people who are genetically susceptible to COPD.

\section{Results}

HHIP risk variant is associated with COPD exacerbations. HHIP variant rs1032296 (risk allele T) has been associated with lung function in smokers (14) and patients with COPD (1). In 6627 non-Hispanic White former and current smokers in the COPDGene Study, we confirmed the COPD risk variant to be associated with COPD $\left(\mathrm{FEV}_{1} / \mathrm{FVC}<0.7\right)$ and lower $\mathrm{FEV}_{1}$. Furthermore, the risk variant was also associated with increased emphysema, peripheral blood WBC counts, and fibrinogen levels (Table 1). As frequent COPD exacerbation is associated with persistent airway and systemic inflammation (15), we examined whether HHIP COPD risk variant is also associated with COPD exacerbation frequency. In a subset of patients with COPD from Table 1 ( $n=3405)$, HHIP risk allele rs1032296 $(\mathrm{T})$ was associated with increased exacerbations in the year prior to enrollment (incidence rate ratio 1.08, $P=0.046$ ) in multivariable analysis (adjusted for age, sex, baseline St. George's Respiratory Questionnaire score, FEV 1 percent predicted, gastroesophageal reflux disease, and genetic ancestry principal components) (Table 2).

Single-cell RNA sequencing of murine lungs identifies expression of Hhip in fibroblasts. Resembling the persistent inflammation in human COPD lungs, prominent lymphoid aggregates are exhibited in Hhip ${ }^{+/-}$mice upon CS exposure and aging $(11,13)$. However, quantitative PCR (qPCR) analysis of sorted lung cell types indicated that Hhip was highly expressed in fibroblasts instead of immune or epithelial cells (Supplemental Figure 1; supplemental material available online with this article; https://doi.org/10.1172/jci.insight.144575DS1). To confirm this and determine molecular impacts of Hhip haploinsufficiency in lungs, we performed single-cell RNA sequencing, primarily using the 10x Chromium (v2) platform in lungs from Hhip ${ }^{+/-}$mice and WT littermates at 4 age points (13) (Figure 1A). These time points represent late development (postnatal 15 days, P15), an early adult stage with normal histology (4 months), middle age with the development of lymphoid aggregates (8 months), and advanced age with emphysema and substantial lymphoid aggregates (11 months). A total of 38,875 cells passing quality control were analyzed (Supplemental Table 1). To account for experimental and technical variability, we used Seurat (version 3.1.4) integrative analysis with SCTransform to identify shared cell states and populations across different data sets and platforms (16) (Supplemental Figure 2). Using graphbased clustering (17), 26 clusters of cells were identified, encompassing all epithelial, mesenchymal, immune (lymphoid and myeloid), and endothelial cell groups (Figure 1, B and C; Supplemental Figure 3A; and Supplemental Data File 1). The graph-based clusters were manually annotated with differentially expressed genes and validated by projection of previously published single-cell RNA-sequencing atlases (18, 19). Additionally, we annotated $\mathrm{T}$ cell subtypes by utilizing the ImmGen database (20), and manually annotated natural killer $\mathrm{T}$ (NKT) cells, which were mostly grouped within regulatory T cells (Supplemental Figure 3, B and C).

Hhip expression was restricted to the mesenchymal population characterized by expression of collagen (Col3a1, Col1a2) and smooth muscle actin (Acta2) and was absent from other clusters, including the immune cell clusters (Figure 1D). These mesenchymal cells were further categorized by subcluster analysis into 9 subclusters by reference-based annotation (19), including matrix fibroblasts, myofibroblasts, lipofibroblasts, and mesothelial population (Figure 1E). Hhip was a top marker gene for the myofibroblast subcluster (AUC 0.81 , adjusted $P<3 \times 10^{-104}$ ), which also expressed gremlin 2, DAN family BMP antagonist (GREM2); ectonucleotide pyrophosphatase/phosphodiesterase (ENPP1); and Acta2 (19) (Figure 1F and Supplemental Data File 1). As expected, Hhip expression was reduced in Hhip ${ }^{+/-}$lung fibroblasts (Figure 1G). 
Table 1. Baseline characteristics of participants (non-Hispanic White former smokers with and without COPD from COPDGene) stratified by the genotype of HHIP variant rs1032296, where T is the COPD risk allele

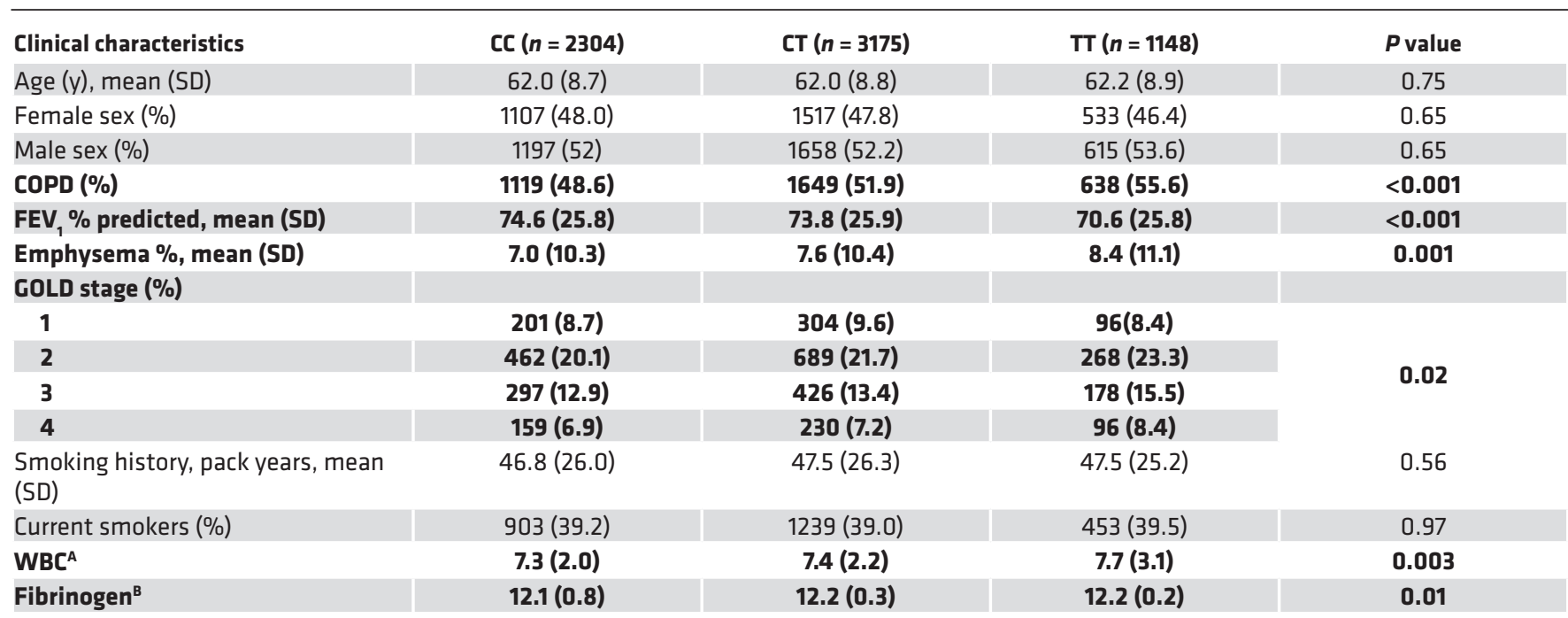

$P$ values are based on Student's 2-tailed $t$ test (where means are reported) or $\chi^{2}$ test for proportions. Bold font indicates significant $P$ value association between HHIP genotype and variables. ASubset of 2756 patients. ${ }^{B}$ Subset of 1023 patients. CC, CT, and TT, 3 different genotypes at rs1032296; FEV forced expiratory volume at 1 second; GOLD, Global Initiative for Chronic Obstructive Lung Disease.

Number of $\mathrm{CD}^{+}$terminal effector cells is increased with aging in lungs from Hhip ${ }^{+/}$mice. Notably, the proportion of $\mathrm{CD}^{+} \mathrm{T}$ cells among lymphoid cells in $\mathrm{Hhip}^{+/-}$mice increased with age (Figure 2), while the relative fraction of $\mathrm{CD}^{+} \mathrm{T}$ cells among lymphoid cells decreased (Supplemental Figure 4), concomitant with the appearance of lung lymphoid aggregates in $\mathrm{Hhip}^{+/-}$mice. Moreover, among CD8 ${ }^{+} \mathrm{T}$ cell subtypes, we found an increase of the percentage of specific $\mathrm{CD}^{+} \mathrm{T}$ cell subgroup marked by expression of killer cell lectin-like receptor G1 (Klrg1) in Hhip ${ }^{+/}$mice at 11 months of age from 3.1\% to $24.5 \%$ while percentage of other lymphoid cell subtypes showed minimal genotypic differences (Figure 2A and Supplemental Figure 4). $\mathrm{K} \operatorname{lrg} 1$ is induced in highly cytotoxic and proliferative $\mathrm{CD} 8^{+} \mathrm{T}$ effector cells and serves as a marker for terminal differentiation of $\mathrm{CD} 8^{+} \mathrm{T}$ cells (21). The expanded $\mathrm{CD} 8^{+} \mathrm{T}$ cell population expressed additional effector T cell markers Cx3cr1, Tbx21, and Gzma but lacked expression of memory T cell markers, such as CD127 (I17r), Cd27, and Tcf7, corresponding to CD8TTEs, or short-lived effector T cells (22) (Supplemental Figure 5A). FACS analysis in lungs from mice at 11 months of age confirmed increased proportion of CD8TTE cells marked by KLRG $1{ }^{\text {hi }} \mathrm{CD} 127^{-}$as well as increased levels of KLRG1 in CD8TTEs in Hhip ${ }^{+/-}$ mice (Figure 2, B and C; and Supplemental Figure 5, B and C).

CD8TTEs have increased expression of Ifny in Hhip ${ }^{+/-}$mice. Cell type-specific differential gene expression analysis showed increased expression of Ifn $\gamma$ in combined $\mathrm{CD} 8^{+} \mathrm{T}$ cells from Hhip ${ }^{+/}$mice (Wilcoxon rank sum test, adjusted $P<0.05$, Figure $3 \mathrm{~A}$ ). We confirmed increased expression of Ifn $\gamma$ in $\mathrm{CD}^{+} \mathrm{T}$ cells from the lungs of 4-month-old $\mathrm{Hhip}^{+/-}$mice by qPCR (Figure 3B). This is most likely driven by CD8TTE, the only immune cell type showing age-dependent increases in Ifn $\gamma$ UMI counts in Hhip ${ }^{+/}$, in contrast to other cell types that can also produce IFN- $\gamma$ (Figure 3A). Furthermore, expression of Tbx21, a key transcription factor inducing expression of Ifn $\gamma$, was increased in Hhip ${ }^{+/-}$CD8TTEs compared with age-matched Hhip H $^{++}$ cells as early as 15 days of age (Figure 3C), suggesting the activation of the Tbx21-Ifn $\gamma$ in Hhip ${ }^{+/}$CD8TTE cells precedes emphysema and lymphocytic aggregates' formation in Hhip ${ }^{+/-}$mice. In contrast, expression of transcription factors important for Th17 (Rorc) and Th2 (Gata3) cell differentiation showed no consistent genotypic difference in a given T cell subtype (Figure 3, D and E).

Pathway analysis on cell type specific gene expression changes suggest fibroblast-immune cell interactions. Cell type-specific pathway analysis on upregulated genes in $\mathrm{Hhip}^{+-}$lungs indicates significant enrichment of inflammatory and immune pathways in fibroblasts and immune cells at as early as 4 months of age, which may drive subsequently increased lymphocytic inflammation with age-associated emphysema in Hhip $^{+/-}$mice at 10 months of age (13) (Supplemental Figure 6 and Supplemental Data File 2). In Hhip ${ }^{+/}$ 
Table 2. HHIP risk variant rs1032296_T is associated with increased COPD exacerbation frequency in the year prior to enrollment

\begin{tabular}{|c|c|c|c|}
\hline Variable & Participants & Incident risk ratio (95\% CI) & $P$ value \\
\hline Exacerbation frequency & $\operatorname{COPD}(n=3405)$ & $1.08(1.00-1.17)$ & $<0.05$ \\
\hline
\end{tabular}

$\mathrm{CD}^{+} \mathrm{T}$ cells, the IFN- $\gamma$ production pathway was enriched (Figure $4 \mathrm{~A}$ ), supporting increased expression of IFN- $\gamma$ (Figure 3, A and B) in $\mathrm{CD}^{+} \mathrm{T}$ cells. Interestingly, upregulated genes in Hhip ${ }^{+/-}$fibroblasts were enriched for pathways related to interaction with lymphocytes, including lymphocyte activation and IL-18 production, an IFN- $\gamma$-inducing cytokine (Figure 4B).

Cell-cell communication, such as fibroblast-CD8 ${ }^{+} \mathrm{T}$ cell crosstalk, commonly occurs through receptor-ligand interaction. Therefore, we further investigated potential genotype-dependent receptor-ligand interactions across multiple cell types using CellPhoneDB (23). We found pervasive genotype-dependent differences in the number of receptor-ligand interactions, starting as early as P15 with changes between fibroblasts and epithelial cells followed by an expansion into other cell type pairs at 8 months of age (Figure 5A and Supplemental Figure 7). For the CD8TEs, the greatest genotypic difference was observed from their interaction with fibroblasts compared with other cell types at 8 months of age (Figure 5A), suggesting fibroblasts as one of the major cell types to modulate the activation of CD8TEs. We then examined the specific receptor-ligand pairs that differed between $\mathrm{CD}^{+} \mathrm{T}$ cells and fibroblasts in Hhip $^{+/-}$mice as compared with $\mathrm{Hhip}^{+/+}$mice. IFN- $\gamma$ and IFN- $\gamma$ receptor interaction between CD8TTEs and fibroblasts was significantly different in Hhip ${ }^{+/-}$mice at as early as 4 months of age (Figure 5B), accompanied by IFN- $\gamma$-induced cytokine-receptor interactions, such as CXCR6-CXCL16 (24). In contrast, such associations were absent for $\mathrm{Hhip}^{+/+}$mice until 11 months of age, indicative of accelerated inflammation in $\mathrm{Hhip}^{+/-}$mice due to fibroblasts-CD8 ${ }^{+} \mathrm{T}$ cells communications at the molecular levels starting as early as 4 months of age, preceding pathological changes (13). On the other hand, Th2-induced cytokine interactions, such as CCR2-CCL11 and TSLP-TSLPR, were absent in hip $^{+/-}$mice, supporting the shift of CD8 $\mathrm{T}$ cells to IFN- $\gamma$-producing type $1 \mathrm{CD}^{+} \mathrm{T}$ cells in Hhip ${ }^{+/-}$mice (Figure $5 \mathrm{~B}$, bottom).

Fibroblast-derived IL-18 enhances IFN- $\gamma$ production in $C D 8^{+} T$ cells from Hhip ${ }^{+-}$mice. The single-cell RNA-sequencing analysis suggested that $\mathrm{Hhip}^{+/-}$lung fibroblasts may interact with and activate CD ${ }^{+}$ $\mathrm{T}$ cells. As genes in the IL-18 production pathway were upregulated in Hhip ${ }^{+/-}$fibroblasts, and IL-18 is a major inducer of IFN- $\gamma$ production in the absence of stimulation of the $\mathrm{T}$ cell antigen receptor in $\mathrm{CD}^{+} \mathrm{T}$ cells $(25,26)$, we hypothesized that secreted factors such as IL-18 from fibroblasts may activate $\mathrm{CD}^{+} \mathrm{T}$ cells. To test this hypothesis, splenic $\mathrm{CD} 8^{+} \mathrm{T}$ cells from 2-month-old WT mice were isolated and treated with conditioned medium (CM) from lung fibroblasts from 11-month-old Hhip $\mathrm{p}^{+/+}$ or Hhip ${ }^{+/-}$mice (Figure 6A). After 24 hours of treatment, increased expression of IFN- $\gamma$ was detected in $\mathrm{CD}^{+} \mathrm{T}$ cells cultured with CM collected from Hhip ${ }^{+/-}$lung fibroblasts (Figure 6B), suggesting that Hhip ${ }^{+/}$lung fibroblast-derived secreted factors are sufficient to drive IFN- $\gamma$ production in $\mathrm{CD}^{+} \mathrm{T}$ cells. Indeed, we found significantly increased 1118 gene expression in Hhip ${ }^{+/-}$lung fibroblasts and increased IL-18 protein levels from Hhip ${ }^{+/-}$fibroblast-derived CM (Figure 6, C and D). In contrast, there was no genotypic difference in the expression of I112a, which is also known to induce IFN- $\gamma$ in T cells (Supplemental Figure 8A). Although IL-18 is highly expressed in alveolar macrophages in the lung (19), we found minimal differences in IL-18 expression in alveolar macrophages from Hhip ${ }^{+/-}$and Hhip ${ }^{+/+}$mice (Supplemental Figure 8B), suggesting that IL-18-derived activation of T cells in Hhip ${ }^{+/-}$lungs is more likely from lung fibroblasts than from macrophages.

Furthermore, knockdown of HHIP with siRNA led to increased expression of IL18 in primary normal human lung fibroblasts and MRC5 lung fibroblast cell line (Figure 6, E and F). IL-18 neutralizing antibody abolished increased expression of Ifn $\gamma$ in $\mathrm{Hhip}^{+/-}$fibroblasts (Figure 6G). These results suggest that HHIP represses IL-18 signaling and that lung fibroblast-derived IL-18, at least partially, contributes to the activation of $\mathrm{CD}^{+} \mathrm{T}$ cells, indicated by increased IFN- $\gamma$ expression.

IL-18 is increased in COPD patients carrying an HHIP risk variant. To determine the relevance of murine findings in patients with COPD, we analyzed the relationship between genotype at the HHIP GWAS 
A
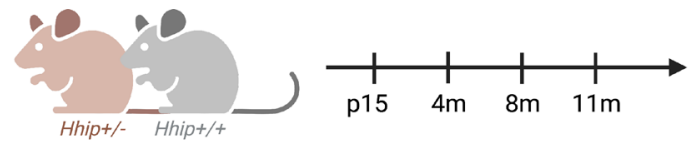

Lung Dissociation

10X Chromium (38k cells)

inDrops (3k cells)

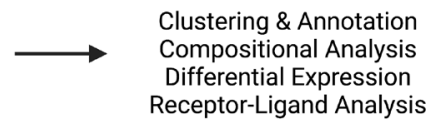

\begin{tabular}{|ccccc|}
\hline Hhipt/- $(\mathrm{n})$ & 2 & 3 & 3 & 2 \\
\hline Lymph.agg & - & - & ++ & +++ \\
emphysema & - & - & - & ++ \\
\hline Hhip+/+ (n) & 2 & 2 & 2 & 2 \\
\hline Lymph.agg & - & - & + & + \\
Emphysema & - & - & - & - \\
\hline
\end{tabular}

B

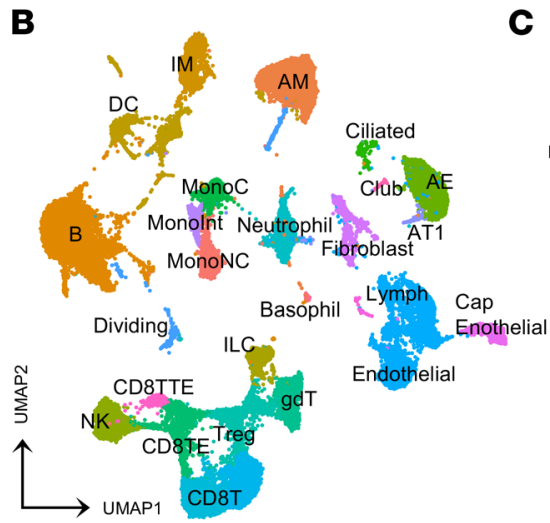

C

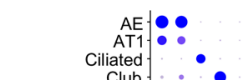

E

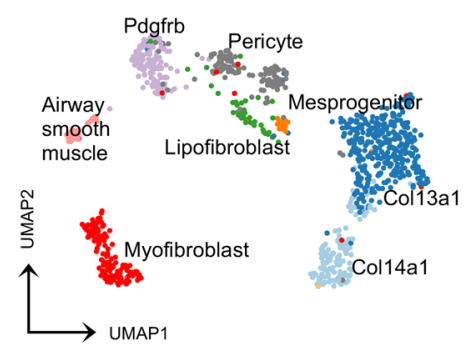

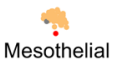$$
\text { F }
$$

Clustering \& Annotation

Receptor-Ligand Analysis
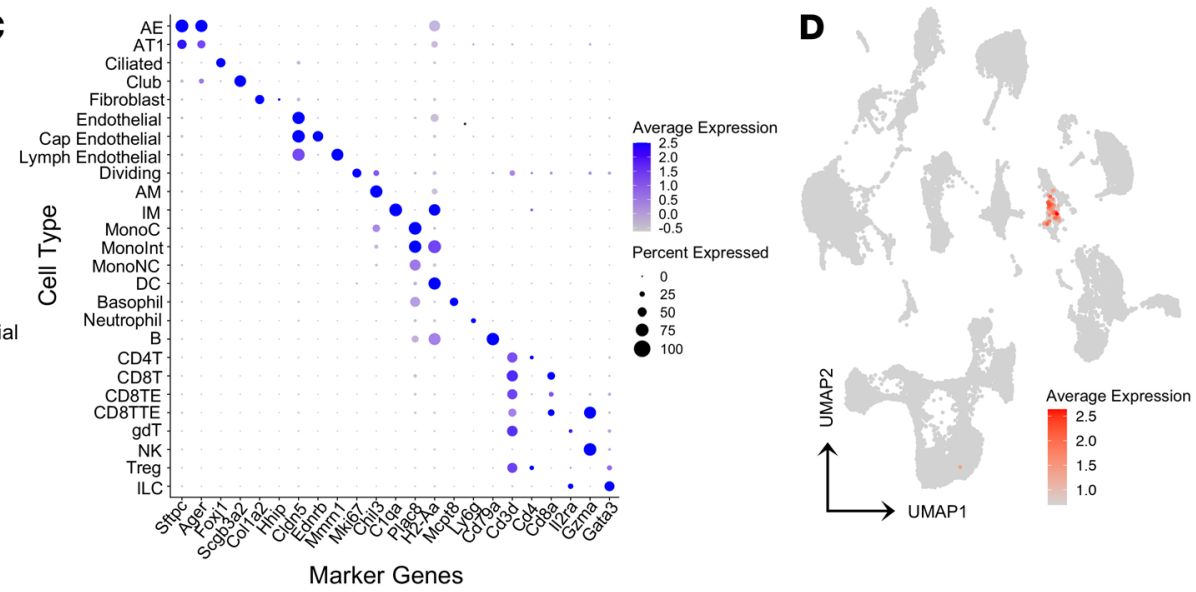

G
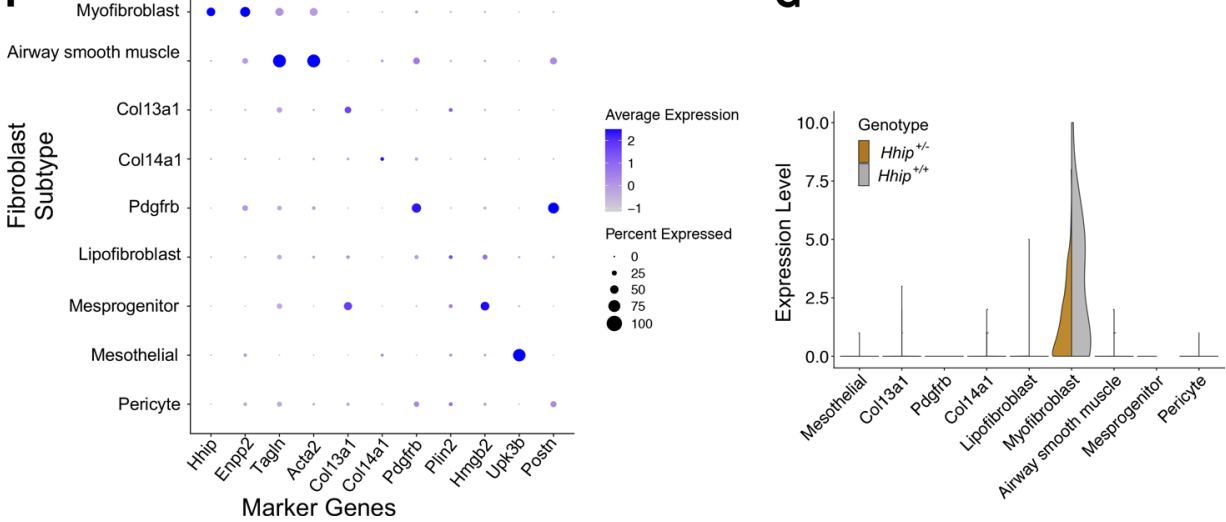

Figure 1. Single-cell RNA sequencing of $\mathrm{Hhip}^{+/-}$and $\mathrm{Hhip}^{+/+}$murine lungs. (A) Experimental design of lung single-cell RNA sequencing from agematched $\mathrm{Hhip}^{+/-}$and WT littermate Hhip ${ }^{+/+}$mice. (B) Unbiased clustering of 38,875 cells from 26 clusters by uniform manifold approximation and projection (UMAP) plot. (C) Expression of representative marker genes across cell type clusters. (D) UMAP plot indicating fibroblast cluster expressing Hhip (red dots). (E) Nine subclusters of lung fibroblasts showing heterogeneity. (F) Expression of representative fibroblast subcluster marker genes shown in dot plot. Hhip is a marker gene for myofibroblast subcluster. (G) Hhip expression is decreased in Hhip ${ }^{+/-}$lungs. Expression values are normalized unique molecular identifier (UMI) count. Lymph.agg, lymphoid aggregates; AE: alveolar epithelial cell; AT1, type 1 alveolar epithelial cell; Cap Endothelial, capillary endothelial cell; Lymph Endothelial, lymphatic endothelial cell; AM, alveolar macrophage; IM, interstitial macrophage; Mesprogenitor: mesenchymal progenitor; MonoC, classical monocyte; Monolnt, intermediate monocyte; MonoNC, nonclassical monocyte; DC, dendritic cell; CD8TE, CD8 ${ }^{+}$effector memory T cell; CD8TTE, CD8+ terminal effector T cell; gdT, $\gamma \delta$ T cell; NK, natural killer cell; Treg, regulatory T cell; ILC, innate lymphoid cell.

locus and blood cytokine profiles in a subset of non-Hispanic White patients from the COPDGene Study $(n=590)$ (Table 3) (27). HHIP risk variant rs1032296 (T) was significantly associated with higher IL-18 levels in serum (log transformed) by multivariable analysis (Table 4). However, no genotypic association with IFN- $\gamma$ was found, possibly reflecting a more complex regulation of IFN- $\gamma$ in blood samples of patients with COPD $(28,29)$. 
A

CD8T effector differentiation

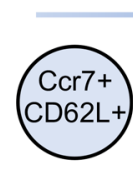

Naïve CD8+ T (CD8T)

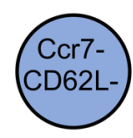

Effector memory (CD8TE)

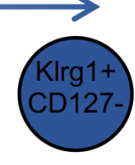

Terminal effector (CD8TTE)

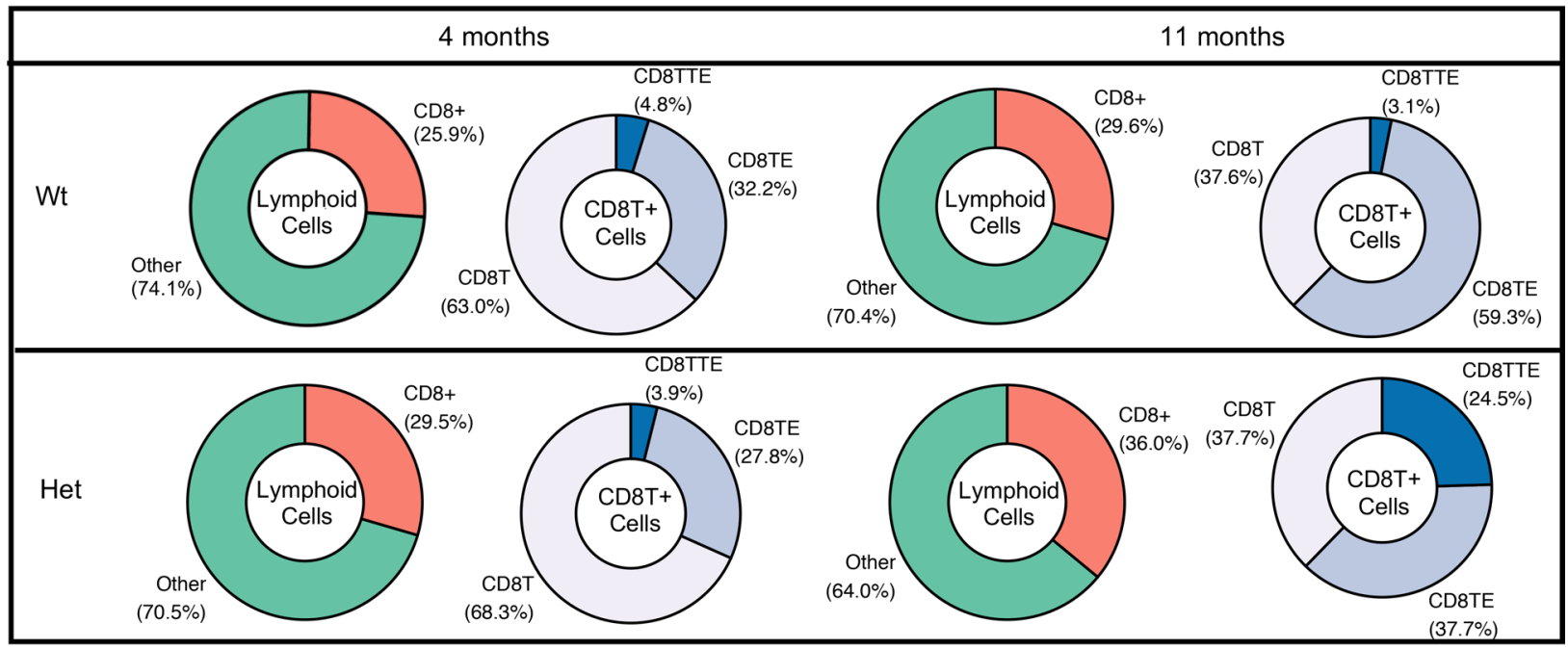

B

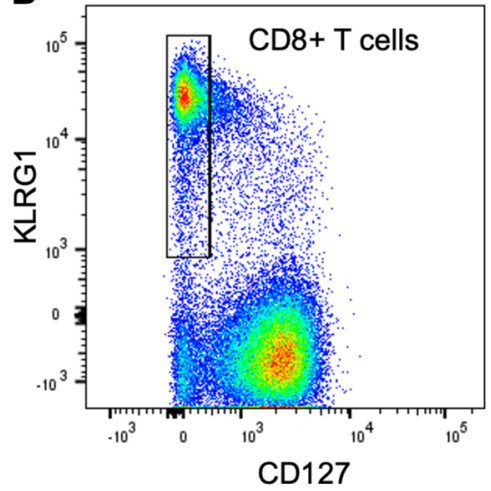

C

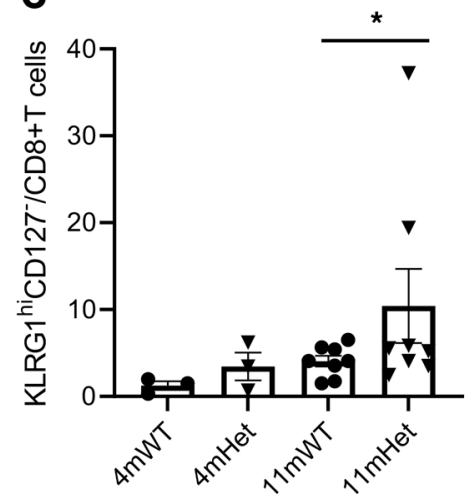

Figure 2. Number of CDTTEs increased in Hhip ${ }^{+-}$mice with age. (A) Naive CD8 ${ }^{+} T$ cells (Ccr7 $\left.{ }^{+} C D 62 L^{+}\right)$differentiate into terminal effector cells (Klrg1 ${ }^{+} \mathrm{CD127}$ ). Proportion of CD8 ${ }^{+} \mathrm{T}$ cells including naive CD8 ${ }^{+} \mathrm{T}$ (CD8T) cells, effector memory CD8 $8^{+} \mathrm{T}$ cells (CD8TE), and terminal effector CD8 ${ }^{+} \mathrm{T}$ cells (CD8TTE) increases with age in Hhip ${ }^{+/}$(Het) mice. (B) FACS gating strategy for CD8TTE cells. CD8 ${ }^{+}$T cells were gated for KLRG1 ${ }^{\text {hiCD127 }}$ populations. (C) Proportion of CD8TTEs in total CD8 ${ }^{+}$T cells. Each point represents an individual biological replicate. Error bars indicate standard error of the mean (SEM). ${ }^{*} P<0.05$. Wilcoxon matched pairs (by age- and sex-matched littermates) signed rank test.

\section{Discussion}

In a human COPD study, we found that the HHIP genetic variant is associated with exacerbation frequency, a key phenotype associated with active inflammation (30). Single-cell RNA sequencing in lungs from the Hhip-haploinsufficient mouse model, which exhibits a similar inflammatory phenotype as seen in patients with COPD even in the absence of smoke exposure, revealed progressive activation of $\mathrm{CD} 8^{+} \mathrm{T}$ cells with age and increased expression of IFN- $\gamma$ preceding emphysematous changes in Hhip ${ }^{+/-}$lungs. Hhip is specifically expressed in lung fibroblasts while absent in $\mathrm{CD}^{+} \mathrm{T}$ cells. Furthermore, fibroblast-CD8 ${ }^{+} \mathrm{T}$ cell interaction and IFN- $\gamma$-inducing pathways are enriched in $\mathrm{Hhip}^{+/-}$fibroblasts, suggesting that Hhip-expressing fibroblasts may repress $\mathrm{CD}^{+} \mathrm{T}$ cell activation, possibly through IL-18, as one of potential mediators. Consistently, the COPD risk allele of the HHIP genetic variant is associated with increased IL-18 levels in patients with COPD.

Among the activated forms of $\mathrm{CD}^{+} \mathrm{T}$ cells, we found a specific increase in $\mathrm{KLRG} 1^{+} \mathrm{CD} 8^{+} \mathrm{T}$ effector cells in Hhip $^{+/-}$mouse lungs. KLRG $1^{+}$CD8TTEs are frequently expanded in response to viral 
A

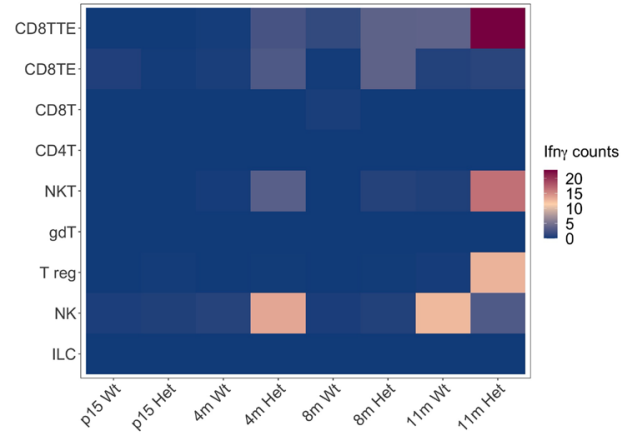

D

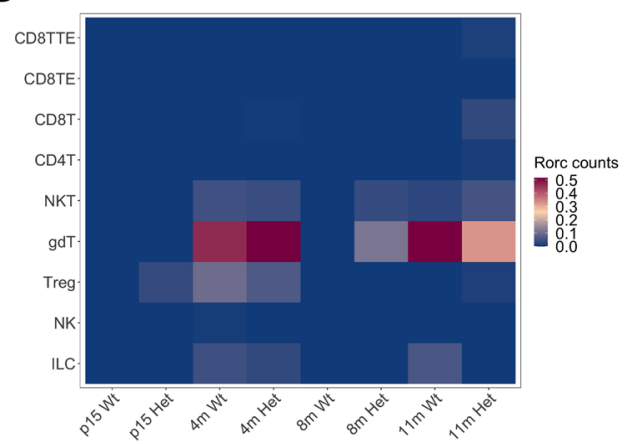

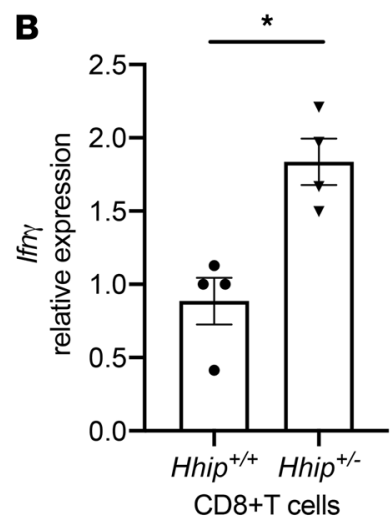

C
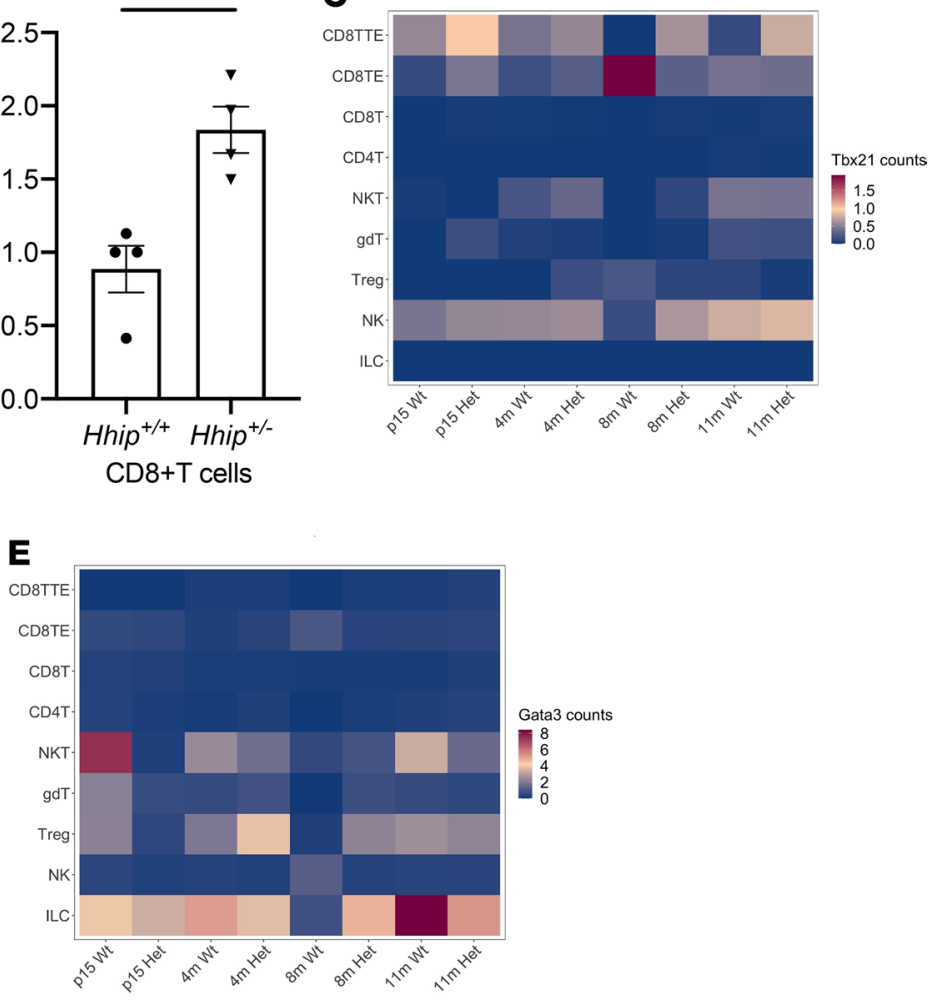

Figure 3. Increased activation of CDTTEs in Hhip/- mice. (A) Average expression (normalized UMI count) of Ifny in various lymphocyte subtypes showing terminal effector CD8 ${ }^{+} \mathrm{T}$ (CD8TTE) cells with increased Ifny expression in Hhip ${ }^{+/-}$mice. (B) Ifny expression is increased in $\mathrm{Hhip}^{+/-}$lung CD8 ${ }^{+} \mathrm{T}$ cells as measured by qPCR ( $n=4$ mice/group, 4 months of age). Error bars indicate SEM. (C) Average expression of Tbx21 in various lymphocytes showing CD8TTE cells with increased Tbx21 expression in Hhip ${ }^{+/-}$mice. (D) Average expression of Rorc in various lymphocyte subtypes. (E) Average expression of Cata3 in various lymphocytes with enrichment in Tregs, NKT cells, and ILCs. ${ }^{*} P<0.05$, unpaired Student's $t$ test. CD8TTE, CD8 ${ }^{+}$terminal effector T cell; CD8TE, CD8 ${ }^{+}$effector memory T cell; CD8T, CD8+ naive T cell; CD4T, CD4+ T cell; NKT, natural killer T cell; gdT, $\gamma \delta$ T cell; Treg, regulatory T cell; NK, natural killer cell; ILC, innate lymphoid cell.

infections and produce IFN- $\gamma(31,32)$ but are also found in patients with autoimmune disease and cancer as dysfunctional $\mathrm{T}$ cells in persistent inflammatory conditions (33).

Both IFN- $\gamma$ and IL-18 signaling pathways are known to contribute to human COPD pathogenesis. First, numbers of IFN- $\gamma$-producing T cells are increased in the lungs of patients with COPD $(34,35)$, and expression of IFN- $\gamma$ by lung CD8 ${ }^{+} \mathrm{T}$ cells also correlates with COPD severity (35). Interestingly, IFN- $\gamma$ recruits inflammatory cells into COPD lungs through upregulation of CXCR3 (28), which also showed increased expression along with CXCL10 in Hhip ${ }^{+-}$lungs at 10 months of age (13). Second, IFN- $\gamma$ induces the release of matrix metalloproteinase-12 (MMP-12) in a transgenic mouse model, leading to inflammation and emphysema $(36,37)$. Indeed, MMP-12 levels are increased in Hhip ${ }^{+/-}$lungs when lung lymphoid aggregates are evident (13). Additionally, IFN- $\gamma$ synergistically acts with TNF- $\alpha$ to induce expression of CXCL10 via NF-кB (38), reinforcing the recruitment of CXCR3/IFN- $\gamma$-expressing T cells to the lung (39). Indeed, TNF- $\alpha$ signaling via NF- $\kappa \mathrm{B}$ pathway was enriched in upregulated genes in Hhip ${ }^{+/-}$lungs (Supplemental Figure 6). Similarly, IL-18, a strong inducer of IFN- $\gamma$, not only promotes emphysema development in murine models $(37,40)$ but also functions as a biomarker and a master cytokine driving COPD progression (41). IL-18 is elevated in COPD patients' lung and sputum samples, which correlates with IFN- $\gamma$ levels (42). Consistent with our findings, stimulation of human lung $\mathrm{CD}^{+} \mathrm{T}$ cells from COPD patients in vitro with IL-18 (plus IL-12) increases IFN- $\gamma$ production, and the expression of IL-18 receptor correlates with spirometrically defined COPD severity (43). Thus, our findings provide one possible mechanism to explain the genetic heterogeneity of augmented inflammation seen in COPD patients.

Whether Hhip regulates IL-18 expression in fibroblasts through its inhibition of the hedgehog pathway requires further study. Overexpression of the hedgehog effector Smoothened in lung mesenchymal cells leads to negligible inflammatory phenotypes in lungs despite evident emphysema (44), suggesting minimal impact of intrinsic hedgehog signaling in lung fibroblasts on the activation of lymphocytes $(18,19,45)$. 
A
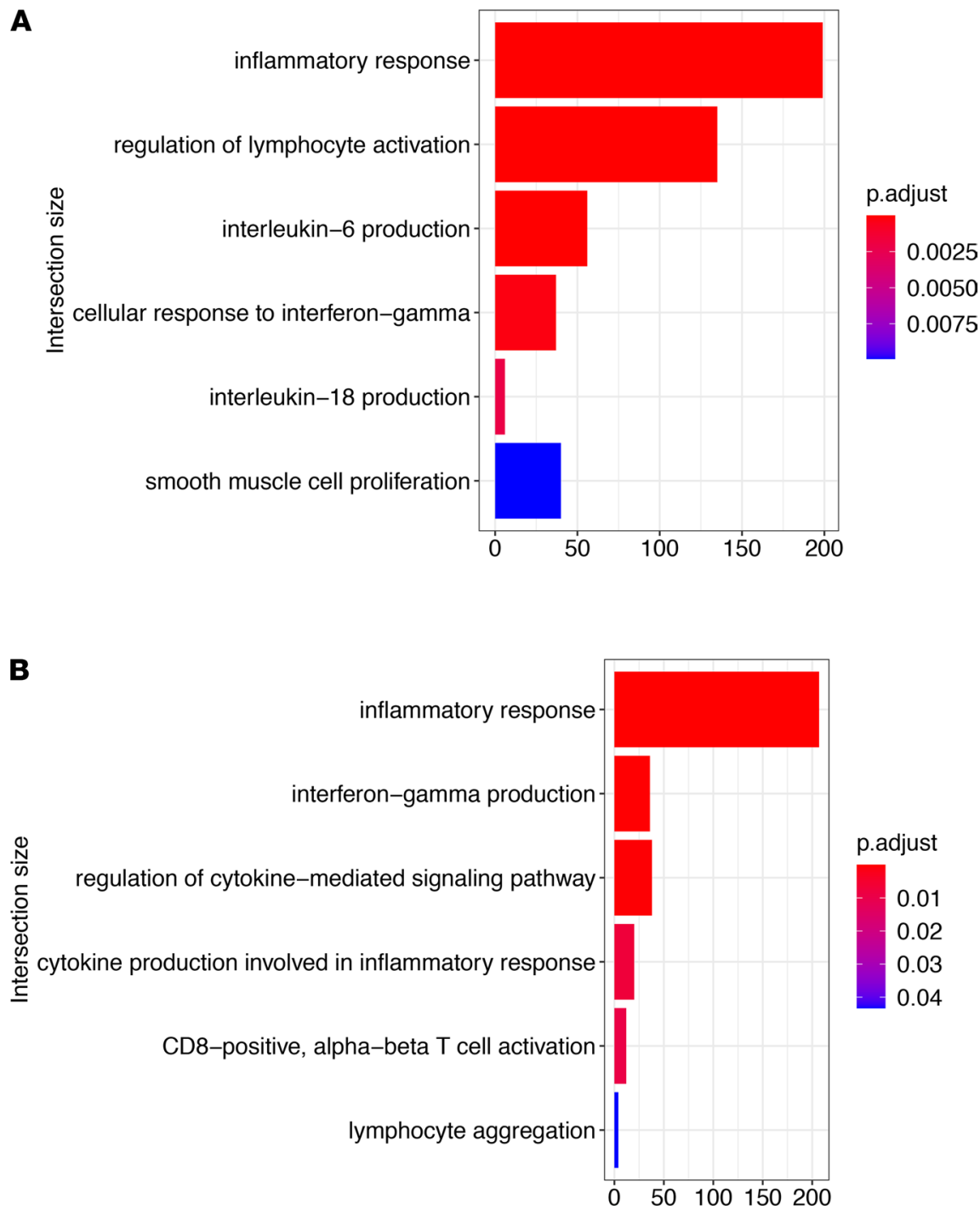

Figure 4. Inflammatory response-related pathways are enriched in $\mathrm{Hhip}^{+/-} \mathrm{CD8}^{+} \mathrm{T}$ cells and fibroblasts. Selected pathways that are significantly enriched with upregulated genes in $\mathrm{Hhip}^{+/-}$compared with $\mathrm{Hhip}^{+/+}$mice by Gene Ontology (CO) analysis in (A) all CD8+ T cells and (B) fibroblasts. Pathway analysis was performed with gprofiler2. $P$ value is FDR adjusted.

Therefore, the molecular mechanism by which HHIP represses IL-18 expression in lung fibroblasts needs future investigation.

Frequent cell-cell communication in lungs, including crosstalk between Hhip-expressing fibroblasts and $\mathrm{CD} 8^{+} \mathrm{T}$ cells, may contribute to the formation of dysfunctional $\mathrm{T}$ cells important for COPD pathogenesis. As shown in Supplemental Figure 7, fibroblasts also had differential receptor-ligand interactions with other cell types, including endothelial cells and ciliated epithelial cells, that were significant as early as P15. Future studies including endothelial and epithelial cells and other lung cell types interacting with fibroblasts will reveal more comprehensive functions of HHIP in lungs through cell-cell communications.

Aside from the contribution of the IL-18/IFN- $\gamma$ axis to such fibroblast-lymphocyte crosstalk, roles of other fibroblast-derived factors need to be assessed in comprehensive cytokine screenings. Furthermore, the association of HHIP genotype with serum IL-18 levels may not be completely attributable to regulation of HHIP on IL-18 expression in lung fibroblasts, which requires further studies. Furthermore, future studies on blocking the lymphocyte inflammation or IL-18/IFN- $\gamma$ signaling to ameliorate the emphysema phenotype in $\mathrm{Hhip}^{+/-}$murine models and examination of expression of IL-18 in human lung tissue are also warranted to pinpoint the therapeutic applications. 
A

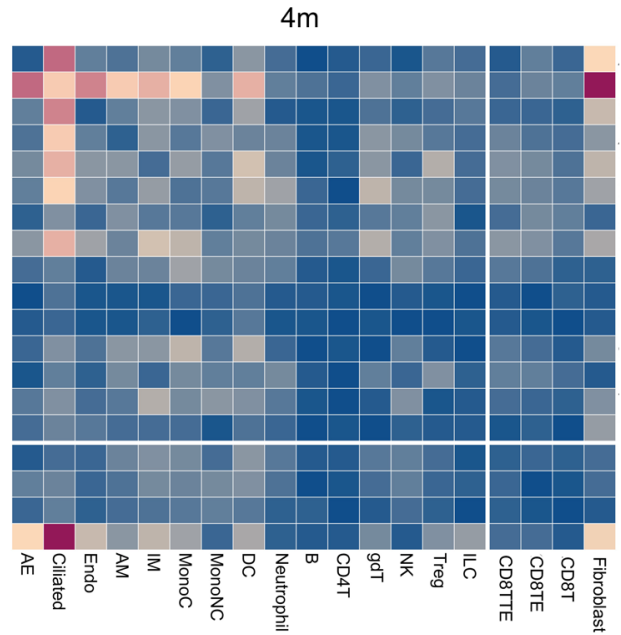

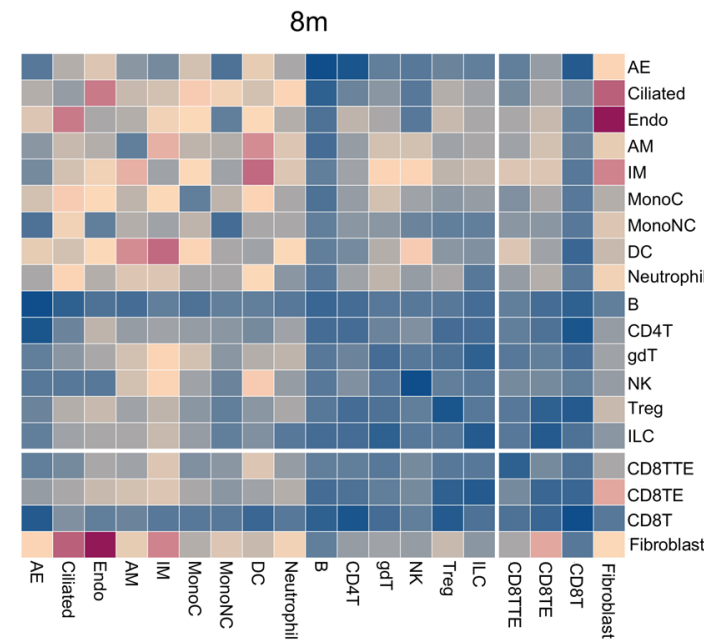

Number of differential interactions

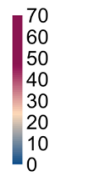

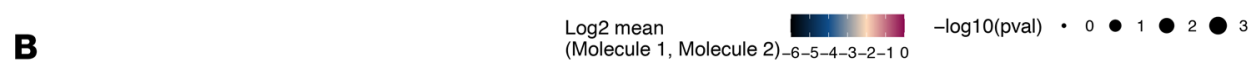

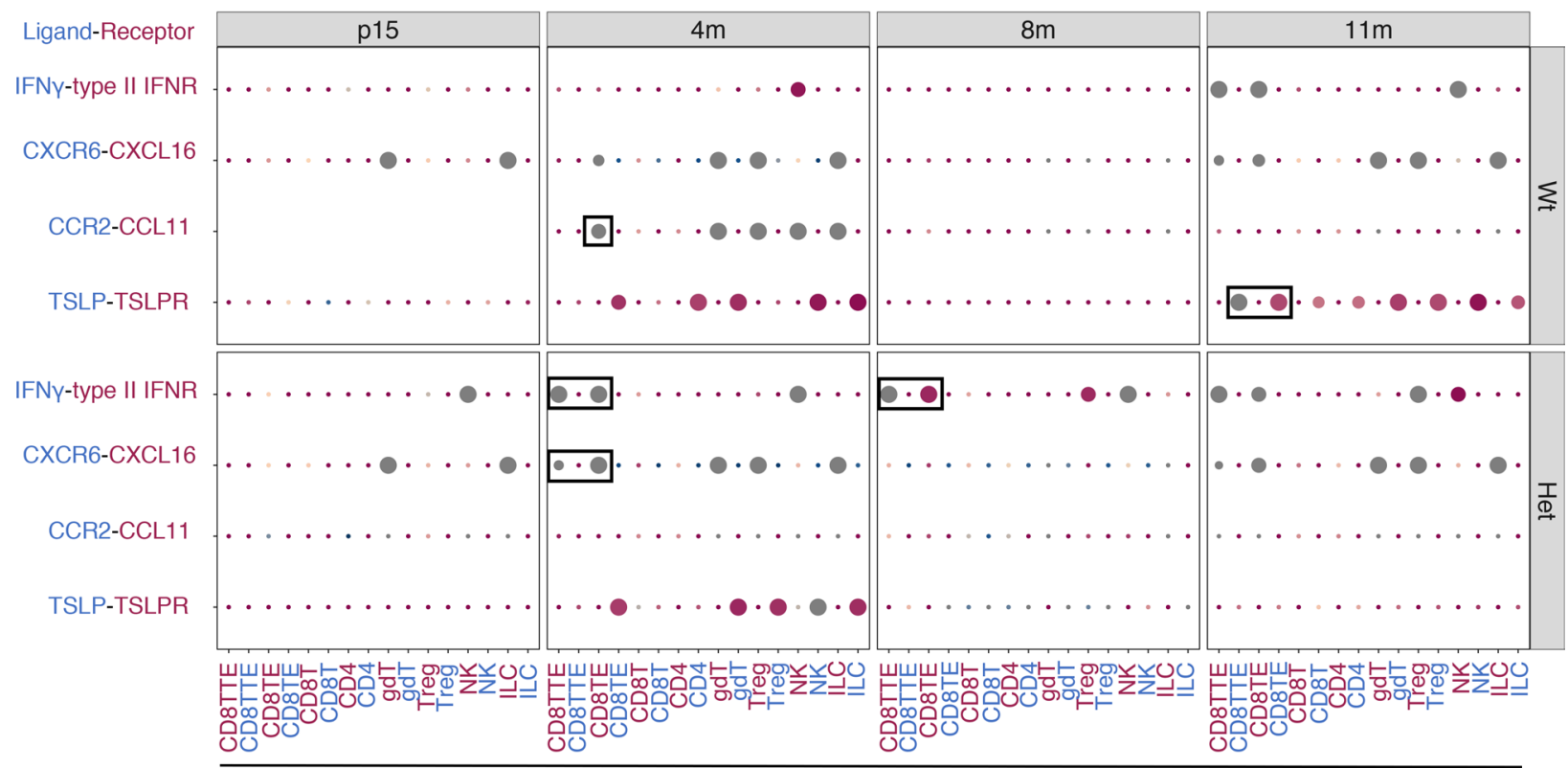

Fibroblast

Figure 5. Receptor-ligand analysis suggests dysregulated fibroblast-T cell interaction in lungs from Hhip-haploinsufficient mice. (A) Genotype-dependent differential receptor-ligand interactions across various cell types from mice at 4 months ( $4 \mathrm{~m})$ and $8 \mathrm{months}$ ( $8 \mathrm{~m})$ of age. Color scale bar indicates number of interaction pairs between cell types. (B) Selected ligand (blue)-receptor (pink) pairs between fibroblasts and immune cells from mice at different age points. Select receptor-ligand interaction pairs that are different by genotype are marked by black rectangles. Dot size indicates $P$ values and the means of expression levels (normalized UMI counts) of interacting molecules are indicated by color of each dot. Wt, $\mathrm{Hhip}^{+/+}$; Het, Hhip ${ }^{+/}$; AE, alveolar epithelial cell; Endo, endothelial cell; AM, alveolar macrophage; IM, interstitial macrophage; MonoC, classical monocyte; MonoNC, nonclasscial monocyte; DC, dendritic cell; B, B cell; CD8T, CD8 ${ }^{+}$naive T cell; CD8TE, CD8 ${ }^{+}$effector memory T cell; CD8TTE, CD8 terminal effector T cell; gdT, $\gamma \delta$ T cell; NK, natural killer cell; Treg, regulatory T cell; ILC, innate lymphoid cell.

Notably, the murine Hhip haploinsufficiency model displays an array of inflammatory phenotypes that resembles human COPD patients, including the peri-airway location of lymphoid aggregates (12), presence of increased $\mathrm{CD} 8^{+} \mathrm{T}$ cells in lymphoid aggregates, and increased number of activated $\mathrm{CD} 8^{+} \mathrm{T}$ cells $(12,13,43)$. The location of these specific inflammatory features coincides with the peribronchial location of Hhip-expressing murine fibroblasts as shown by Tsukui and colleagues (46), and the observation of IL-18 induction in human adult lung fibroblasts upon silencing of HHIP suggests a plausible role of the mesenchymal HHIP in the inflammatory pathophysiology of COPD. However, recent single-cell 
A

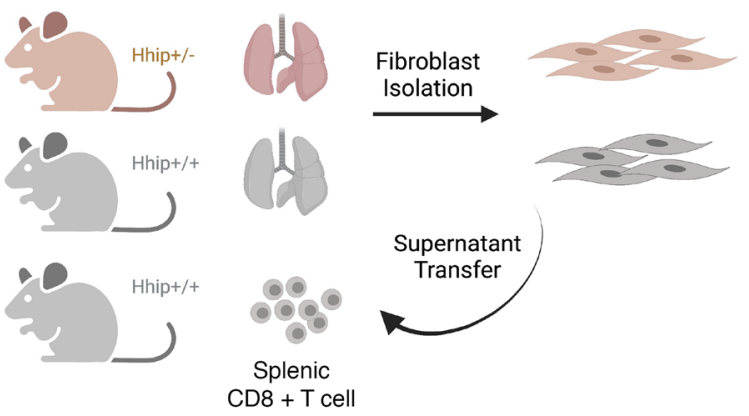

B

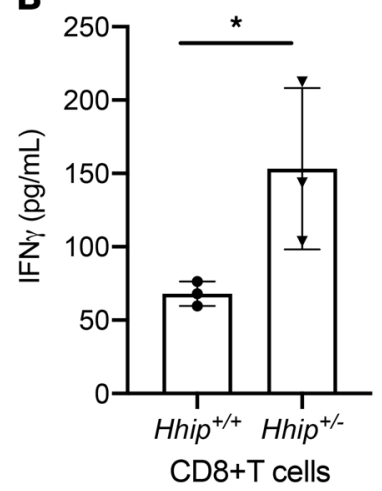

C

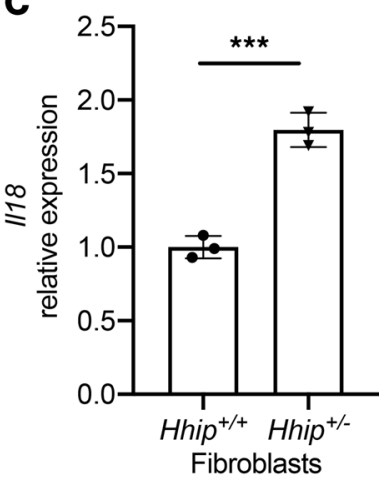

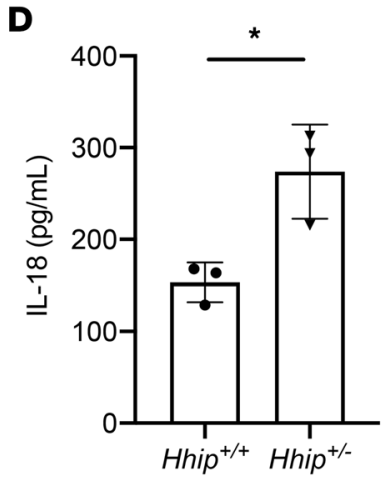

Conditional Medium

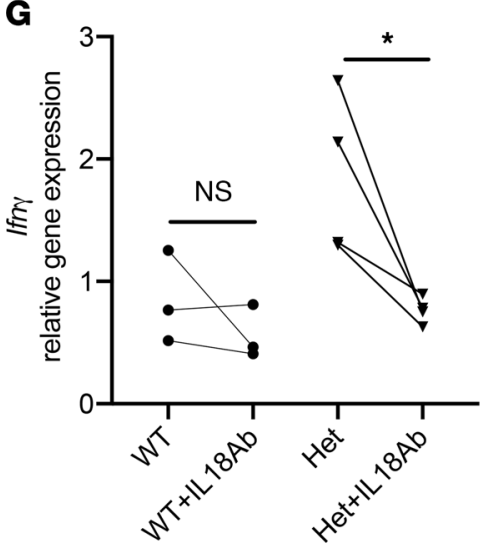

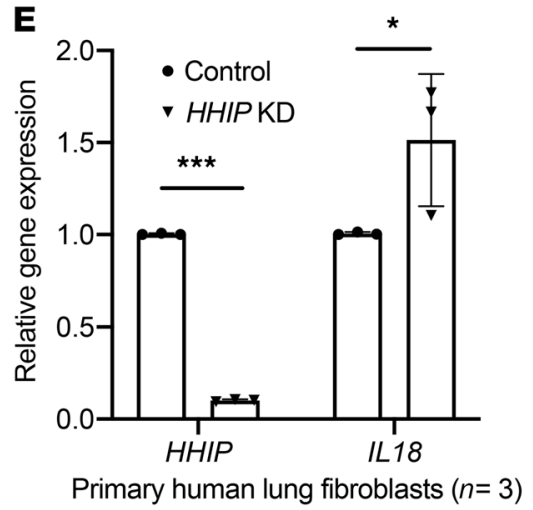

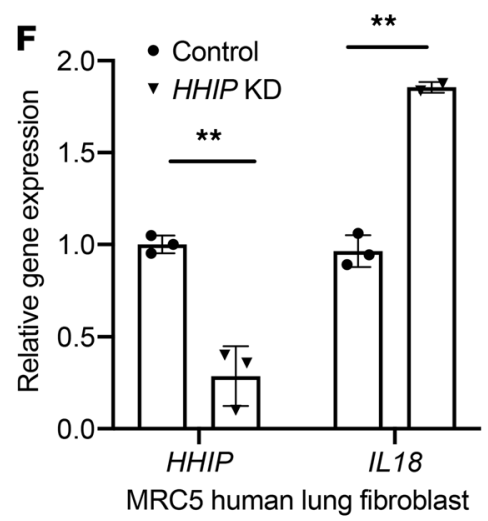

Figure 6. Hhip $^{+/-}$lung fibroblasts induce increased IFN- $\gamma$ production from CD8+ $\mathrm{T}$ cells. (A) Experimental design. Splenic CD8 ${ }^{+} \mathrm{T}$ cells from $\mathrm{Hhip}^{+/+}$mice were treated with conditioned medium (CM) from lung fibroblasts from 11-month-old $\mathrm{Hhip}^{+/+}$and $\mathrm{Hhip}^{+/-}$mice for 24 hours. (B) WT CD8 ${ }^{+} \mathrm{T}$ cells incubated with $\mathrm{Hhip}^{+/-}$-derived CM produced increased levels of IFN- $\gamma$. (C) Lung fibroblasts from $\mathrm{Hhip}^{+/-}$mice have higher expression of I/18. (D) IL-18 level is elevated in the Hhip ${ }^{+/-}$-derived CM measured by ELISA. (E) Knockdown of HHIP by siRNA in adult primary human lung fibroblasts $(n=3)$ and (F) human fetal lung fibroblast cell line MRC5 led to increased expression of I/18. (G) Expression of If $n \gamma$ in WT splenic CD8 ${ }^{+} \mathrm{T}$ cells treated with conditioned medium derived from $\mathrm{Hhip}^{+/+}$or $\mathrm{Hhip}^{+/-}$fibroblasts for 24 hours, with or without IL-18 neutralizing $A b(30 \mu \mathrm{g} / \mathrm{mL})$ as measured by qPCR with Cd8a as the reference gene. Each point represents an individual biological replicate. Data are represented as mean \pm SD, with $n \geq 3$ per group. ${ }^{*} P<0.05$, ${ }^{* *} P<0.01$, ${ }^{* *} P<0.001$, unpaired Student's $t$ test (B-F), paired Student's $t$ test $(\mathbf{G})$. Representative results are shown (from $\geq 2$ replicated experiments with 2-4 mice in each repeat for B-D and F). KD, knockdown.

RNA sequencing expression studies in adult human lungs suggest additional alveolar expression of HHIP (46-48). Specifically, although we find that HHIP expression is restricted in the fibroblast population in mouse lungs (Figure 1, D and E), as shown in other publications $(19,46,48)$, as well as neonatal human lungs (49), strong HHIP expression was also found in a subset of type 2 alveolar epithelial cells in adult rat, pig, and human lungs (47). These observations raise questions such as the long-term effect of HHIP expression in the developing lung mesenchyme, and the role of HHIP in type 2 alveolar epithelial cells to the airway inflammation, which requires further investigation in future studies. Taken together, further studies with conditional ablation of HHIP in adult tissues and cell type-specific ablation of HHIP in human lung organoid models or species with both epithelial and mesenchymal expression of HHIP, such as rats, would elucidate the developmental, compartment-specific effect of HHIP in COPD pathogenesis.

In sum, our study provides the utility of single-cell transcriptomics in inferring cell type-specific gene expression and intercellular interactions and suggests a potential mechanism by which the genetic factor HHIP may control lymphocytic inflammation in COPD. Additionally, receptor-ligand analysis using enriched single-cell RNA-sequencing data provided cell-cell communication roadmaps in murine lungs from mice at 4 different ages. COPD exacerbations are treated with antiinflammatory, immunomodulatory 
Table 3. Baseline characteristics of study participants with IL-18 measurements (non-Hispanic Whites from COPDGene) stratified by HHIP risk variant rs1032296_T

\begin{tabular}{|c|c|c|c|c|}
\hline Clinical characteristics & CC $(n=215)$ & CT $(n=278)$ & $\mathrm{TT}(n=97)$ & $P$ value \\
\hline Age, mean (SD) & $63.1(8.8)$ & $63.8(8.4)$ & $64.5(8.3)$ & 0.42 \\
\hline Sex (female) (\%) & $98(45.6)$ & $137(49.3)$ & $51(52.6)$ & 0.49 \\
\hline Smoking history (pack years), mean (SD) & $47.6(29.9)$ & $46.2(23.5)$ & $50.6(27.2)$ & 0.38 \\
\hline COPD (\%) & $117(54.4)$ & $165(59.4)$ & $68(70.1)$ & 0.03 \\
\hline $\mathrm{FEV}_{1}(\mathrm{~mL})$, mean $(\mathrm{SD})$ & $2.08(1.08)$ & $1.99(0.99)$ & $1.86(0.96)$ & 0.21 \\
\hline IFN- $\gamma$, mean (SD) & $4.00(2.8)$ & $3.64(1.9)$ & $3.87(2.0)$ & 0.2 \\
\hline
\end{tabular}

Bold font indicates significant $P$ value association. $P$ values are based on Student's 2-tailed $t$ test (where means are reported) or $\chi^{2}$ test for proportions.

drugs, such as azithromycin (50), with incompletely defined mechanism of actions. With comprehensive gene signatures in multiple immune cell types in lungs associated with age-dependent emphysema, our work may ultimately facilitate the development of novel immunomodulatory therapies in COPD.

\section{Methods}

\section{Human patients}

Details of the COPDGene Study (Genetic Epidemiology of COPD, ClinicalTrials.gov NCT00608764) have been described previously (27). The COPDGene Study enrolled 10,192 smokers with and without COPD between November 2007 and April 2010. Patients were clinically stable, with at least 30 days since their last exacerbation. Of the 10,300 participants (including 108 lifelong nonsmokers), 6884 (67\%) were non-Hispanic White (NHW) participants, of whom 6646 (97\%) had genotype data available for HHIP variant rs1032296. We analyzed $6627 \mathrm{NHW}$ patients with lung function information and a subset of 3405 patients with COPD defined as the postbronchodilator ratio of $\mathrm{FEV}_{1} / \mathrm{FVC}<0.7$. Genotyping was performed using HumanOmniExpress array (Illumina) (2). Blood cytokines were measured in a subset of patients $(n=590)$ as previously published (51). Briefly, blood biomarkers were measured by customized 13-panel multiplex assays (Myriad-RBM) (51). Measurement results for IL-18 and IFN- $\gamma$ were used for analysis.

Mice

$H_{i p^{+/-}}$mice, as described previously, were generated by replacing exon 1 of Hhip with a lacZ reporter gene (52) followed by backcrossing to C57BL/6J background (11). Hhip ${ }^{+/-}$mice and littermate WT Hhip ${ }^{+/+}$, a total of 18 , with matching age and sex were harvested at different ages for single-cell RNA sequencing and in vitro experiments.

\section{Generation of lung single-cell suspension}

For harvesting, mice were euthanized by $\mathrm{CO}_{2}$ asphyxiation followed by exsanguination. Lungs were perfused with chilled PBS via the right atrium. For lung cells' isolation without enrichment (15 out of 18 samples), dispase (50 U/mL) was instilled into lungs, which were dissected and minced on ice into approximately $1 \mathrm{~mm}^{3}$ cubes. Lungs were digested using collagenase/dispase for 30 minutes at $37^{\circ} \mathrm{C}$, then passed through $70 \mu \mathrm{m}$ and $40 \mu \mathrm{m}$ cell strainers. Cells were centrifuged at $300 \mathrm{RCF}$ for 5 minutes, then incubated with RBC lysis buffer. Cells were stained with trypan blue for viability assessment, which showed greater than $85 \%$ viability with this method. The single-cell suspension was sorted by FACS for DAPI-negative viable cells using MoFlo Astrios EQ (Beckman Coulter).

For enriched CD45-negative lung cells (two 4-month samples and one 8-month sample), lungs were digested using a mouse lung isolation kit with gentleMACS dissociator (Miltenyi Biotec), then were passed through $70 \mu \mathrm{m}$ and $40 \mu \mathrm{m}$ cell strainers. After centrifugation at $300 \mathrm{RCF}$ for 5 minutes, cells were incubated with RBC lysis buffer. Cells were then stained with DAPI, DRAQ5 (Abcam), and 
Table 4. HHIP variant rs1032296 (T) is associated with IL-18 levels in serum samples from COPDGene participants

\begin{tabular}{l|c|c|c}
\hline Variable & Participants & Estimate (95\% CI) & P value \\
\hline IL-18 & All participants $(n=590)$ & $1.03(1.00-1.05)$ & $<0.05$ \\
\hline
\end{tabular}

Multivariable linear regression adjusted for age, sex, current smoking status, and genetic ancestry principal components. IL-18 value was log-transformed.

CD45-PE Ab (BioLegend) and selected for DAPI-negative, DRAQ5-positive, and CD45-negative cells using On-chip Sort cell sorter (On-chip Biotechnologies) (53).

\section{Single-cell RNA sequencing}

Single-cell RNA sequencing using 10x Chromium. Lung single-cell suspension was loaded onto a 10x Chromium Single Cell instrument per manufacturer's instructions. cDNA amplification and library construction were performed according to the 10x Chromium Single Cell 3' v2 manufacturer's protocol. Quality control of the library was performed by Agilent Bioanalyzer and qPCR by using an Illumina Library Quantification Kit (KAPA Biosystems, KK4824). Libraries were sequenced on the Illumina HiSeq (Harvard Biopolymers Facility) or NovaSeq (Novogene) platform, using paired-end reads, with the following read length: 26 bp read 1 for cell barcode and UMI, 8 bp i7 index for sample index, and 98 bp for transcript.

Single-cell RNA sequencing using inDrops. For 8-month-old mouse samples, the single-cell suspension was directly loaded into a microfluidic device. Cells were encapsulated with barcoded hydrogel microspheres, reverse transcriptase, and lysis reagents. Primers were photo-cleaved by UV exposure after encapsulation as previously described (54). cDNA amplification and library construction (v3) were performed at the Single Cell Sequencing Core at Harvard Medical School. Indexed libraries were pooled and sequenced on an Illumina NextSeq 500 (Harvard Bauer Facility), using paired-end reads, with the following read length: $61 \mathrm{bp}$ for transcript, $14 \mathrm{bp}$ for barcode and UMI, 8 bp i7 index for part of barcode, and $8 \mathrm{bp}$ i5 index for sample index.

\section{Cell culture studies}

Cell line. Human fetal lung fibroblast cell line MRC5 (ATCC, CCL-171) was cultured in DMEM supplemented with $10 \%$ fetal bovine serum (FBS) and antibiotics in a humidified incubator at $37^{\circ} \mathrm{C}$ with $5 \% \mathrm{CO}_{2}$.

Primary cell isolation and culture. Primary mouse lung fibroblasts were isolated by culturing the enzymatically digested lungs with liberase (MilliporeSigma) in DMEM/F12 containing 15\% FBS in a humidified incubator at $37^{\circ} \mathrm{C}$ and $5 \% \mathrm{CO}_{2}$. Fibroblasts were passaged and cultured in DMEM supplemented with $10 \%$ FBS before being used for experiments. Cells were used between passages 1 and 2 .

Primary mouse alveolar type 2 epithelial cells were isolated as previously described (55); briefly, lung was enzymatically digested with dispase. Macrophages were removed by incubation with biotinylated antiCD45, anti-TER-119, and anti-CD16/32 antibodies (clones 30-F11, TER-119, and 2.4G2, BD Biosciences) and then subjected to magnetic selection with streptavidin-conjugated magnetic beads (Invitrogen). Cells were seeded onto Petri dishes precoated with mouse IgG (I5381, MilliporeSigma) and cultured for 2 hours at $37^{\circ} \mathrm{C}$. Nonadherent alveolar epithelial cells were removed from IgG plates and resuspended in DMEM/F-12 medium supplemented with $10 \%$ FBS.

$\mathrm{CD}^{+} \mathrm{T}$ lymphocytes from mouse lungs and spleens were isolated by negative selection using $\mathrm{CD} 8 \mathrm{a}^{+} \mathrm{T}$ Cell Isolation Kit (Miltenyi Biotec).

Alveolar macrophages were isolated by performing bronchoalveolar lavage (total $10 \mathrm{~mL}$ ), and purity of alveolar macrophage (>90\%) was confirmed by Diff Quick staining (MilliporeSigma) after cytospin preparation.

Splenocytes were prepared by excising the spleen and passing the cells into a $40 \mu \mathrm{m}$ cell strainer.

Primary human lung fibroblasts were isolated from the lung tissues of healthy individuals (Marsico Lung Institute, University of North Carolina at Chapel Hill, North Carolina). The lung tissue was cut into small pieces and seeded onto culture dishes at $37^{\circ} \mathrm{C}$ in a humidified $5 \% \mathrm{CO}_{2} /$ air incubator. The dishes were placed upside down for 2 hours, then turned back, and DMEM was added. The primary human lung fibroblasts grew to $90 \%$ confluence within 2 to 3 weeks for subsequent experiments. 
Immunofluorescence staining and flow cytometry. Lung $\mathrm{CD}^{+} \mathrm{T}$ cells were washed in PBS containing $0.5 \%$ bovine serum albumin and $2 \mathrm{mM}$ EDTA, then incubated with the following antibodies: CD127 (A7R34) PE (eBioscience 12-1271-81), Klrg1 (2F1) APC (eBioscience 17-5893-81), CD4 (RM4-5) PerCP-Cy5.5 (eBioscience 45-0042-82), Tcr $\beta$ BV605 (BioLegend 109241), CD45.2 BV510 (BioLegend 109837), and Cd8a BV711 (BioLegend 100747). Measurements were performed on a FACSymphony flow cytometer (BD), and analysis was performed with FlowJo software.

Fibroblast-derived supernatant transfer. On day 1, fibroblasts from 11-month-old mice were plated in 12 -well culture plates at $5 \times 10^{5}$ cells/well in DMEM with $10 \%$ FBS. On day $2,1 \times 10^{6}$ fresh splenic $\mathrm{CD}^{+} \mathrm{T}$ cells harvested from 2-month-old $\mathrm{Hhip}^{+/+}$mice were cultured in supernatant collected from Hhip $^{+/+}$or Hhip ${ }^{+/-}$fibroblasts with the addition of anti-CD3 and anti-CD28 Ab $(0.25 \mu \mathrm{g} / \mathrm{mL}$ each, 562163,553294 , BD Biosciences). $\mathrm{CD}^{+} \mathrm{T}$ cells were maintained at $37^{\circ} \mathrm{C}$ with $5 \% \mathrm{CO}_{2}$ for an additional 24 hours before subsequent qPCR analysis. In experiments for Figure 6G, IL-18 neutralizing Ab (30 $\mu \mathrm{g} /$ $\mathrm{mL}, \mathrm{D} 048-3$, R\&D Systems) was added to CD8 ${ }^{+} \mathrm{T}$ cells for 24 hours on day 2 before cell collection for quantitative real-time PCR analysis.

RNA interference. We transfected siRNAs targeting human HHIP and nontargeting control pools (J-01301809-0010, D-001810-10-05, respectively, Dharmacon) into primary human lung fibroblasts using Lipofectamine RNAi/MAX (Invitrogen). The culture medium was changed to a normal medium 2 hours after transfection.

\section{Quantitative reverse transcription PCR}

Total RNA from cells was isolated with RNeasy Mini Kit (QIAGEN) according to the manufacturer's protocol. A total of $100 \mathrm{ng}$ of RNA was reverse-transcribed to cDNA with High-Capacity cDNA Reverse Transcription Kit (Life Technologies). For qPCR, TaqMan gene expression assays were used on a QuantStudio 12K Real-Time PCR system (Applied Biosystems). FAM-conjugated TaqMan probes for Hhip, Ifn $\gamma$, Il18, Il12a, Il12b, Gapdh, Cd8a, and Tbp (mouse) and HHIP, IL18, and GAPDH (human) (IDT) were used with TaqMan Gene Expression Master Mix (Applied Biosystems). Gene expression values were normalized to GAPDH for isolated fibroblasts and MRC5 cell lines and to Cd8a and Tbp for isolated CD8 ${ }^{+} \mathrm{T}$ lymphocytes and macrophages. Expression levels of target genes were calculated based on the $2^{-\Delta \Delta \mathrm{Ct}}$ method. For undetermined $\mathrm{Ct}$ values, $\mathrm{Ct}$ value of 40 was used to calculate relative expression level.

\section{ELISA}

Supernatant from mouse or human fibroblasts was analyzed for IL-18 levels using the Mouse IL-18 Platinum ELISA (Invitrogen, BMS618-3) or Human Total IL-18/IL-1F4 Quantikine ELISA Kit (R\&D Systems). IFN- $\gamma$ levels in cell culture media from $\mathrm{CD}^{+} \mathrm{T}$ lymphocytes were analyzed using Mouse IFN- $\gamma$ ELISA MAX Standard (BioLegend) according to the manufacturer's protocol.

\section{Single cell RNA-sequencing data analysis}

$10 x$ data preprocessing. Sequencing output was demultiplexed by the Cell Ranger (version 2.0.0) pipeline. The number of cells in each sample was estimated by the Cell Ranger software (Estimated Number of Cells). LacZ transgene mapping was performed by incorporating LacZ sequence to the $\mathrm{mm} 10$ reference genome to confirm the genotypic identity of $\mathrm{Hhip}^{+/-}$mice.

inDrops data preprocessing. Sequencing output was demultiplexed by the inDrops pipeline. To exclude potential doublets and empty cells, we filtered cells with UMI counts over 3000 or less than 200, as well as cells with mitochondrial percentage over 15\% (Supplemental Table 1). After constructing the raw count matrix, we used the R package Seurat (v3) (56) for data analysis.

Seurat. Library count matrices were processed with variance stabilizing transformation (SCTransform workflow) in Seurat v3.1.4. The fraction of mitochondrial UMI counts per cell were regressed out. Utilizing the Pearson residuals by the variance stabilizing transformation of libraries, all library count matrices were integrated using a reference library ( $4 \mathrm{~m} \mathrm{Het}-2$ ) that had the highest sequencing depth per cell. The reference-based integration did not differ in a biologically meaningful fashion from the full pairwise comparison suggested by the standard workflow. For the anchor finding and integration, 30 dimensions were used. Compared with simple merge of count matrices, batch effect was mainly driven by cell capture technology (inDrops vs. 10x), sequencing platform and/or preparation (HiSeq, NextSeq vs. NovaSeq; whole lung cells vs. On-chip-enriched CD45-negative cells). This batch effect was effectively removed after the integration procedure was assessed by the merge of clusters from 3 batches in the UMAP embedding plot. 
The integrated Pearson residual matrix was used to cluster cells. Specifically, principal component analysis generated 40 principal components that were subsequently used for neighborhood graph construction, UMAP embedding, and cluster identification (Leiden algorithm, algorithm $=4$ for FindClusters function in Seurat v3.1.4). The choice of the number of principal components (PCs) was determined partially by the SD of each PC as assessed by Elbowplot function in Seurat v3.1.4. The overall embedding and clustering were not sensitive by the choice of PC numbers. To cluster all cells, we used a resolution parameter of 1.5. For $\mathrm{T}$ cell and fibroblast population, subclustering was necessary to identify detailed subtypes (NKT cells and fibroblast subtypes), as increases in PCs or resolution parameters were ineffective in distinguishing subtle differences.

To annotate cluster identities objectively, published single-cell data annotation from a mouse cell atlas (18) were transferred to our data for annotation of lung structural cell types using TransferData function in Seurat v3.1.4. Subsequently, the graph-based clusters from the Leiden algorithm were subjected to marker identification by Wilcoxon rank sum test using the wilcoxauc function of the presto package (1.0.0) (57), utilizing the Pearson residuals from the variance stabilizing transformation of the Seurat workflow. Together with the initial label from the mouse atlas, the markers were manually inspected to assign the overall annotation for each graph-based cluster. For downstream analysis, several graph-based clusters were merged with a broader term (dendritic cells, B cells, endothelial cells, and alveolar epithelial cells), in particular if they were shown as a continuum in the UMAP embedding plot. The T cell cluster ( $C d 3 a^{+}$cell population) and NK and ILC populations that formed a continuum in the UMAP embedding plot were subjected to further marker identification by pairwise comparison of adjacent clusters and subtype identification by the ImmGen DataBrowser (Gene Skyline, ImmGen ULI RNASeq) (58), to compare for specific markers for reference populations. Final main clustering resulted in 26 cell (sub)types from 44 clusters (resolution $=1.5$ ). Note that a few PCs were enriched for cell cycle genes, and 439 cells were grouped mainly based on these PCs and estimated to be in G2M and S phase based on the CellCycleScoring function in Seurat v3.1.4. Neither removing the cell cycle gene-enriched PC, nor regressing out the scores of S phase and G2M phase derived from the CellCycleScoring function, could merge the cells into original cell type clusters, suggesting that these cells had insufficient information to be incorporated into the respective cell type clusters. Given that these cells did not have obvious bias in terms of genotype and age to the entire population, and the majority of $\mathrm{S}$ and G2M phase estimated cells were still found in cell type clusters, no further attempt to reassign the 439 cells (annotated as "Dividing") into subtype annotation was made.

In the $C d 3 a^{+}$cell population group, NKT cell population was not clearly assigned as a graph-based subcluster, likely because of the very close transcriptomic profile to related cell clusters, suggested by the close overlap of scores from the R package singleR (20) with ImmGen reference database (58). Thus, the T cell, NK, and ILC cluster was subsetted. To minimize batch effect from technical platform and cell capture method, we grouped the cells based on batches and performed the integration procedure after variance stabilizing transformation was conducted. Subclustering was performed using 20 PCs with Leiden algorithm with resolution of 0.8 .

For fibroblast subclustering, we conducted the same approach as described above by grouping the subsetted libraries into batches (inDrops, CD45-negative enriched 10x, whole lung 10x) and integration. Subclustering was performed using 10 PCs with Leiden algorithm with resolution of 0.8. We compared the individual fibroblast subclusters with published data (Gene Expression Omnibus, GEO: GSE104154) (19) using TransferData function in Seurat v3.1.4. Before the label transfer, the original count matrix from the Xie et al. data set (21) was processed in a similar fashion, using variance stabilizing transform and 40 PCs. The annotation matched well with the newly processed cluster and embedding diagram. Transferred labels also matched well to the graph-based clusters. Note that for the myofibroblast label, a distinct, small smooth muscle cell cluster was also labeled, given that the Xie et al. data set did not contain this cell type.

Cell type-specific differential gene expression analysis was performed on Pearson residuals from the SCTransform in Seurat (59). We applied Wilcoxon rank sum test from presto package; differential expression was defined as log fold change $>0.2$ and adjusted $P$ (FDR corrected $)<0.05$.

Average expression was calculated by natural log transformation of averaged expression of RNA assay using AverageExpression function in Seurat.

Receptor-ligand interaction. Receptor-ligand interaction analysis was performed by CellPhone DB python package (23). Orthologous human gene names replaced mouse gene symbols using the bioMart $\mathrm{R}$ package (60). Counts from nonorthologous genes were discarded prior to analysis. The normalized count data 
originally processed in the total UMI counts were used for the analysis. The data were split into genotype and age for individual processing, and 1000 iterations were used for $P$ value calculation. Statistically significant interactions between cell types for each age/genotype group were compared, and the number of interactions that differed between genotypes were counted and shown in the heatmap from mice at each age. Statistically significant interactions among cell types with genotypic differences from mice at each age were shown as receptor-ligand pairs in a dot plot.

Functional enrichment analysis. GO term enrichment was performed using g:Profiler (61).

Data availability. Genomic data for the manuscript are available from GEO GSE149843 (https://www. ncbi.nlm.nih.gov/geo/query/acc.cgi?acc=GSE149843).

\section{Statistics}

Nongenomic experimental data were analyzed using a 2-tailed Student's unpaired $t$ test unless otherwise stated, using GraphPad Prism software. Student's $t$ test with Welch's correction was performed when group sizes were not equal. $P<0.05$ was considered significant.

Human genotype analysis. Genetic analysis of human patients was performed using additive genetic models. Negative binomial regression was used to assess HHIP genotype association with COPD exacerbation frequency in the year prior to enrollment adjusted for genetic ancestry PCs, age, sex, baseline St. George's Respiratory Questionnaire score, $\mathrm{FEV}_{1}$ percent predicted, and gastroesophageal reflux disease.

Linear regression was used to evaluate HHIP genotype association with log-transformed IL-18 and IFN- $\gamma$ adjusted for genetic ancestry PCs, age, sex, and current smoking status. All analyses were performed using R. All $P$ values were 2 sided, and less than 0.05 was considered statistically significant.

\section{Study approval}

All animal studies were approved by the Institutional Animal Care and Use Committee of Brigham and Women's Hospital.

COPDGene (ClinicalTrials.gov NCT00608764) is a multicenter, observational study (27). Institutional review board approval was obtained at each of the 21 participating clinical centers, and written informed consent was obtained from all participants.

\section{Author contributions}

JHY and XZ designed the study. JHY performed single-cell RNA sequencing. JHY and CL performed data analysis. JHY, TL, and SL performed in vitro assays and $\mathrm{PPCR}$. SX maintained mice colonies and performed genotyping. JHY, CL, TL, EYK, JLC, LP, RBP, EKS, CPH, and XZ interpreted data and edited the manuscript.

\section{Acknowledgments}

We thank Scott Randell (University of North Carolina, Chapel Hill, North Carolina, USA) for providing normal human lung tissues. We thank Harvard Medical School Single Cell Core for inDrops processing. We thank Elizabeth Henske (Brigham and Women's Hospital, Harvard Medical School, Boston, Massachusetts, USA) for the use of gentleMACS dissociator.

Funding sources were K08HL146972 to JHY, R01HL127200 and R01 148667 to XZ, R01HL137927 and R01HL147148 to XZ and EKS, I01 CX000911 to JLC, and R00HG008399 to LP. The COPDGene project is supported by U01 HL089897 and U01 HL089856 from the NIH National Heart, Lung, and Blood Institute. The content is solely the responsibility of the authors and does not necessarily represent the official view of the National Heart, Lung, and Blood Institute or the NIH. COPDGene is also supported by the COPD Foundation through contributions made to an Industry Advisory Board that has included AstraZeneca, Bayer, Boehringer Ingelheim, Genentech, GlaxoSmithKline, Novartis, Pfizer, Siemens, and Sunovion.

Address correspondence to: Xiaobo Zhou, Brigham and Women's Hospital, EBRC 620c, 221 Longwood Ave., Boston, Massachusetts 02115, USA. Phone: 617.525.7866; Email: Xiaobo.Zhou@channing.harvard.edu. 
1. Lutz SM, et al. A genome-wide association study identifies risk loci for spirometric measures among smokers of European and African ancestry. BMC Genet. 2015;16(1):138.

2. Cho MH, et al. Risk loci for chronic obstructive pulmonary disease: a genome-wide association study and meta-analysis. Lancet Respir Med. 2014;2(3):214-225.

3. Hobbs BD, et al. Genetic loci associated with chronic obstructive pulmonary disease overlap with loci for lung function and pulmonary fibrosis. Nat Genet. 2017;49(3):426-432.

4. Pillai SG, et al. A genome-wide association study in chronic obstructive pulmonary disease (COPD): identification of two major susceptibility loci. PLoS Genet. 2009;5(3):e1000421.

5. Cho MH, et al. A genome-wide association study of emphysema and airway quantitative imaging phenotypes. Am J Respir Crit Care Med. 2015;192(5):559-569.

6. Boueiz A, et al. Genome-wide association study of the genetic determinants of emphysema distribution. Am J Respir Crit Care Med. 2017;195(6):757-771.

7. van der Plaat DA, et al. Genome-wide association study on the FEV1/FVC ratio in never-smokers identifies HHIP and FAM13A. J Allergy Clin Immunol. 2017;139(2):533-540.

8. Repapi E, et al. Genome-wide association study identifies five loci associated with lung function. Nat Genet. 2010;42(1):36-44.

9. Zhou X, et al. Identification of a chronic obstructive pulmonary disease genetic determinant that regulates HHIP. Hum Mol Genet. 2012;21(6):1325-1335.

10. Chuang P-T, et al. Feedback control of mammalian Hedgehog signaling by the Hedgehog-binding protein, Hip1, modulates Fgf signaling during branching morphogenesis of the lung. Genes Dev. 2003;17(3):342-347.

11. Lao T, et al. Haploinsufficiency of Hedgehog interacting protein causes increased emphysema induced by cigarette smoke through network rewiring. Genome Med. 2015;7(1):12.

12. Hogg JC, et al. The nature of small-airway obstruction in chronic obstructive pulmonary disease. NEngl J Med. 2004;350(26):2645-2653.

13. Lao T et al. Hhip haploinsufficiency sensitizes mice to age-related emphysema. Proc Natl Acad Sci U S A. 2016;113(32):E4681-E4687.

14. Wain LV, et al. Novel insights into the genetics of smoking behaviour, lung function, and chronic obstructive pulmonary disease (UK BiLEVE): a genetic association study in UK Biobank. Lancet Respir Med. 2015;3(10):769-781.

15. Perera WR, et al. Inflammatory changes, recovery and recurrence at COPD exacerbation. Eur Respir J. 2007;29(3):527-534.

16. Butler A, et al. Integrating single-cell transcriptomic data across different conditions, technologies, and species. Nat Biotechnol. 2018;36(5):411-420.

17. Satija R, et al. Spatial reconstruction of single-cell gene expression data. Nat Biotechnol. 2015;33(5):495-502.

18. Han X, et al. Mapping the mouse cell atlas by microwell-seq. Cell. 2018;172(5):1091-1107.

19. Xie T, et al. Single-cell deconvolution of fibroblast heterogeneity in mouse pulmonary fibrosis. Cell Rep. 2018;22(13):3625-3640

20. Aran D, et al. Reference-based analysis of lung single-cell sequencing reveals a transitional profibrotic macrophage. Nat Immunol. 2019;20(2):163-172.

21. Buchholz VR, et al. T cell fate at the single-cell level. Annu Rev Immunol. 2016;34:65-92.

22. Herndler-Brandstetter D, et al. KLRG $1^{+}$effector CD8 ${ }^{+} \mathrm{T}$ cells lose KLRG1, differentiate into all memory T cell lineages, and convey enhanced protective immunity. Immunity. 2018;48(4):716-729.

23. Efremova M, et al. CellPhoneDB: inferring cell-cell communication from combined expression of multi-subunit ligand-receptor complexes. Nat Protoc. 2020;15(4):1484-1506.

24. Abel S, et al. The transmembrane CXC-chemokine ligand 16 is induced by IFN-gamma and TNF-alpha and shed by the activity of the disintegrin-like metalloproteinase ADAM10. J Immunol. 2004;172(10):6362-6372.

25. Yang J, et al. Induction of interferon-gamma production in Th1 CD4+ T cells: evidence for two distinct pathways for promoter activation. Eur J Immunol. 1999;29(2):548-555.

26. Dinarello CA, et al. Interleukin-18 and IL-18 binding protein. Front Immunol. 2013;4:289

27. Regan EA, et al. Genetic epidemiology of COPD (COPDGene) study design. COPD. 2011;7(1):32-43.

28. Grumelli S, et al. An immune basis for lung parenchymal destruction in chronic obstructive pulmonary disease and emphysema. PLoS Med. 2004;1(1):075-083.

29. Takabatake N, et al. Impaired systemic cell-mediated immunity and increased susceptibility to acute respiratory tract infections in patients with COPD. Respir Med. 2005;99(4):485-492.

30. Barnes PJ. Inflammatory endotypes in COPD. Allergy. 2019;74(7):1249-1256.

31. Henson S, et al. KLRG-1 signalling induced defective Akt (ser473) phosphorylation and proliferative dysfunction of highly differentiated CD8+ T cells. Blood. 2009;113(26):6619-6628.

32. Cui W, Kaech SM. Generation of effector CD8+ T cells and their conversion to memory T cells. Immunol Rev. 2010;236(1):151-166

33. Li L, et al. KLRG1 restricts memory T cell antitumor immunity. Oncotarget. 2016;7(38):61670-61678.

34. Saetta M, et al. CD8+ T-lymphocytes in peripheral airways of smokers with chronic obstructive pulmonary disease. Am J Respir Crit Care Med. 1998;157(3 pt 1):822-826.

35. Freeman CM, et al. CC chemokine receptor 5 and CXC chemokine receptor 6 expression by lung CD8+ cells correlates with chronic obstructive pulmonary disease severity. Am J Pathol. 2007;171(3):767-776.

36. Wang Z, et al. Interferon gamma induction of pulmonary emphysema in the adult murine lung. J Exp Med. 2000;192(11):1587-1600

37. Kang MJ, et al. IL-18 induces emphysema and airway and vascular remodeling via IFN- $\gamma$, IL-17A, and IL-13. Am J Respir Crit Care Med. 2012;185(11):1205-1217.

38. Hardaker EL, et al. Regulation of TNF-alpha- and IFN-gamma-induced CXCL10 expression: participation of the airway smooth muscle in the pulmonary inflammatory response in chronic obstructive pulmonary disease. FASEB J. 2004;18(1):191-193.

39. Barnes PJ, Cosio MG. Characterization of T lymphocytes in chronic obstructive pulmonary disease. PLoS Med. 2004;1(1):e20.

40. Hoshino T, et al. Pulmonary inflammation and emphysema: role of the cytokines IL-18 and IL-13. Am J Respir Crit Care Med. 2007;176(1):49-62.

41. Dima E, et al. Implication of Interleukin (IL)-18 in the pathogenesis of chronic obstructive pulmonary disease (COPD). Cytokine. 2015;74(2):313-317. 
42. Briend E, et al. IL-18 associated with lung lymphoid aggregates drives IFN $\gamma$ production in severe COPD. Respir Res. 2017;18(1):159.

43. Freeman CM, et al. Cytotoxic potential of lung CD8+ T cells increases with chronic obstructive pulmonary disease severity and with in vitro stimulation by IL-18 or IL-15. J Immunol. 2010;184(11):6504-6513.

44. Wang C, et al. Expansion of hedgehog disrupts mesenchymal identity and induces emphysema phenotype. J Clin Invest. 2018;128(10):4343-4358.

45. Schaum N, et al. Single-cell transcriptomics of 20 mouse organs creates a Tabula Muris. Nature. 2018;562(7727):367-372.

46. Tsukui T, et al. Collagen-producing lung cell atlas identifies multiple subsets with distinct localization and relevance to fibrosis Nat Commun. 2020;11(1):1920.

47. Raredon MSB, et al. Single-cell connectomic analysis of adult mammalian lungs. Sci Adv. 2019;5(12):eaaw3851.

48. Travaglini KJ, et al. A molecular cell atlas of the human lung from single-cell RNA sequencing. Nature. 2020;587(7835):619-625.

49. Ardini-Poleske ME, et al. LungMAP: the molecular atlas of lung development program. Am J Physiol Lung Cell Mol Physiol. 2017;313(5):L733-L740.

50. Barnes PJ, et al. Chronic obstructive pulmonary disease. Nat Rev Dis Primers. 2015;1:15076.

51. Sun W, et al. Common genetic polymorphisms influence blood biomarker measurements in COPD. PLoS Genet. 2016;12(8):e1006011.

52. Chuang PT, McMahon AP. Vertebrate Hedgehog signalling modulated by induction of a Hedgehog-binding protein. Nature. 1999;397(6720):617-621.

53. Watanabe M, et al. A novel flow cytometry-based cell capture platform for the detection, capture and molecular characterization of rare tumor cells in blood. J Transl Med. 2014;12(1):143.

54. Klein AM, et al. Droplet barcoding for single-cell transcriptomics applied to embryonic stem cells. Cell. 2015;161(5):1187-1201.

55. DeMaio L, et al. Characterization of mouse alveolar epithelial cell monolayers. Am J Physiol Lung Cell Mol Physiol. 2009;296(6):L1051-L1058.

56. Stuart T, et al. Comprehensive integration of single-cell data. Cell. 2019;177(7):1888-1902.

57. Korsunsky I, et al. Presto scales Wilcoxon and auROC analyses to millions of observations [preprint]. https://doi. org/10.1101/653253. Posted on bioRxiv May 29, 2019.

58. Heng TSP, et al. The Immunological Genome Project: networks of gene expression in immune cells. Nat Immunol. 2008;9(10):1091-1094

59. Hafemeister C, Satija R. Normalization and variance stabilization of single-cell RNA-seq data using regularized negative binomial regression. Genome Biol. 2019;20(1):296.

60. Durinck S, et al. BioMart and Bioconductor: a powerful link between biological databases and microarray data analysis. Bioinformatics. 2005;21(16):3439-3440.

61. Raudvere U, et al. g:Profiler: a web server for functional enrichment analysis and conversions of gene lists (2019 update). Nucleic Acids Res. 2019;47(w1):W191-W198. 